\title{
电子全息及其在材料科学中的一些应用
}

\author{
田焕芳 李建奇 ${ }^{*}$ \\ (中国科学院物理研究所先进材料与结构分析研究部, 北京 100080. * 联系人, E-mail: 1jq@aphy.iphy.ac.cn)
}

\begin{abstract}
摘要 Gabor 在 20 世纪 40 年代就提出了电子全息干涉成像的基本原理, 随着相干场发射电子枪的发展, 到现在为止, 电子全息技术已经获得了长足的发展和广泛的应用. 本文评述了电子全息的发展、基本原 理以及在磁性、超导和异质结等材料学领域中的一些应用, 对这一研究技术的末来发展进行了简单 论述.
\end{abstract}

关键词 电子全息 电子双棱镜 相位 振幅 界面 磁畴

随着电子显微学的发展, 电磁透镜球差的存在 不可避免地限制了电子显微镜的分辨率. 20 世纪 40 年代的电子显微镜技术还不能精确地在原子水平上 研究物质的结构, 为突破电子显微镜的分辨极限, 矫 正电磁透镜的球差, Gabor 在 1948 年提出了干涉成像 的方法，并且命名为“全息”，拉丁语的意思是“whole writing”. 实际上这种电子全息技术对光源的相干性 要求很高, 普通的光源很难获得干涉花样. 20 世纪 50 年代到 70 年代电子全息的研究主要集中在 Tübingen 大学, Möllenstedt, Düker 和 Wahl 领导的研究小组开 展这项工作. 这段时期激光的出现提供了较好的相 干光源, Michigan 大学的 Leith 等人开展的光学全息 获得了极大的发展和应用. 但直到 20 世纪 70 年代场 发射电子枪的出现, 电子全息才获得突破性的发展, 推动了整个电子显微学的发展, 其应用范围也大大 扩展.

除了提高分辨极限的设计思想外，电子全息最 重要的应用是能够直观地给出穿过样品的入射电子 波的相位变化, 与传统的高分辨电子显微学成像不 同. 传统的高分辨电子显微像是利用散射电子束与 透射电子束的相位差来产生衬度的, 高分辨电子显 微学最主要的问题在于成像过程中丢失了电子波的 相位信息，而这一信息可以通过电子全息术的方法 获得. 本文中我们主要介绍电子全息的基本工作原 理、发展及其在材料科学(磁性和非磁性材料)中的一 些应用。

电子全息有 20 多种实现方式 ${ }^{[1]}$, 既有在TEM下 的同轴、离轴、明场和暗场模式，又有在STEM下的 操作模式. 所采用的双棱镜也已经从获得广泛发展 和应用的单电子双棱镜，逐渐发展到两个甚至三个 双棱镜研究的开发和应用. 本文主要介绍当前应用
较为广泛的离轴电子全息成像方法, 这种方法比较 简单且易在TEM模式下实现.

\section{1 电子全息的基本工作原理}

电子波与物质相互作用后会产生相位变化, 全 息术是利用物体所产生的菲涅耳衍射与相干本底叠 加形成的干涉, 所得到的全息图并不像物体, 但包含 了重现物体所必需的全部信息: 振幅和相位. 它是利 用衍射现象获得物体像的方法, 并不需要用透镜成 像. 与传统的透镜成像过程完全不同, 电子全息术可 以将出射电子波函数的相位和振幅用底片或 $\mathrm{CCD}$ 等 记录介质全部记录下来(电子全息图), 通过对电子全 息图的重构可以将出射电子波函数的相位和振幅分 离开, 分别得到单独的相位衬度像和振幅衬度像.

电子全息术成像分两步完成: (i) 穿过样品的 电子波(物波)与穿过真空的电子波(参考波)形成的相 干本底发生干涉, 并记录在底片或 $\mathrm{CCD}$ 上获得全息 图. (ii) 用一束单色光沿平行于参考波的方向照射 全息图, 重现物体的像. 物体像的重构有光学重构和 数字重构两种方法, 目前基本上都采用数字重构, 用 一个软件包的形式来实现, 但是不同的实验室根据 需要可能采用不同的软件包.

电子全息成像的几何光路图如图 1(a) ${ }^{[2]}$ 所示. 由 场发射电子枪发出的电子波分成两束, 一束在真空 中传播, 另一束则穿透样品传播, 这两束光在经过透 镜之后与穿过样品之前可以看作两个虚点光源穿过 样品, 如图 1(b) $)^{[3]}$ 所示. 在样品下表面由于双棱镜的 干涉作用, 使样品下表面的出射电子波和真空中参 考波向光轴方向偏转, 在重叠部分发生干涉, 从而在 像平面上形成电子全息图. 双棱镜是一根直径约为 $0.6 \mu \mathrm{m}$ 的二氧化硅细丝，表面蒸镀了一层金, 与一个 直流电源相连, 可以进行 $180^{\circ}$ 旋转调节. 电源的电压 


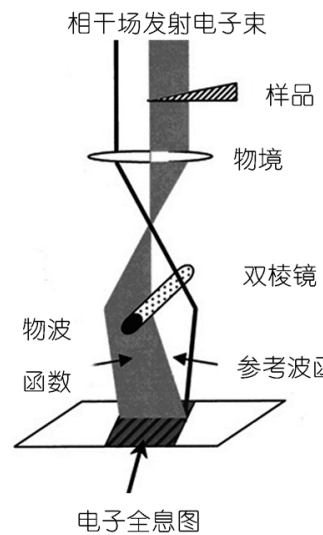

(a)

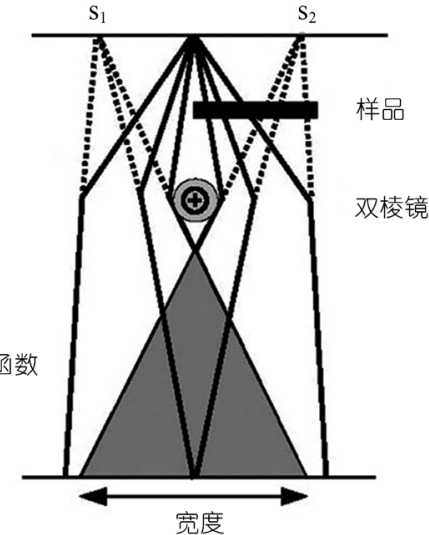

(b)

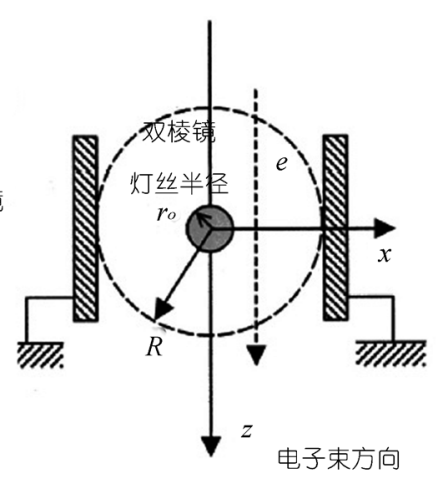

(c)

图 1 离轴电子全息形成示意图

(a) 电子全息形成光路图; (b) 电子波经过双棱镜时的光路示意图; (c) 双棱镜示意图

调节范围一般为 $0 \sim 500 \mathrm{~V}$, 且极性可以反转. 在二氧 化硅细丝的两侧各有一片接地的金属板, 使二氧化 硅与金属板之间形成静电场，如图 1(c) $)^{[2]}$ 所示.

双棱镜既可以放在样品和物镜之间，也可以放 在成像透镜之后, 当静电双棱镜放在样品与物镜之 间时，来自样品较大区域的散射波可以参与干涉，可 获得较多的样品信息, 适合获得高放大倍数的全息 图. 当静电双棱镜处于物镜之后, 参与相干衍射的电 子波来自相对较小的区域, 适合获得较低倍数的全 息图. 散射波和参考波可看成是从两个虚光源发出 的, 它们的位置可通过改变物镜励磁电流来调节. 大 部分静电双棱镜位于虚光源以下, 当静电双棱镜位 于虚光源以下时, 它对地的极性应该是正电压, 当静 电双棱镜位于虚光源以上时, 它对地应为负电压. 当 前常用的场发射枪电子显微镜的静电双棱镜均是安 装在选区光阑位置, 这样根据需要调节和固定起来 比较容易. Harada等人 ${ }^{[4]}$ 采用两个双棱镜成像, 大大 提高了相位分辨率.

\section{1 重构基本理论}

透射电子显微镜中电子光源可以用平面波函数 来代替, 波函数可以表示为

$$
\phi_{p}=\mathrm{e}^{\mathrm{i} \theta_{p}}=\mathrm{e}^{\mathrm{i} k r},
$$

为简单起见, 振幅看作 $1, \theta_{p}$ 为相位, $\boldsymbol{k}$ 为电子束的波 矢 $(\boldsymbol{k}=2 \pi / \lambda, \lambda$ 为电子波的波长 $), r$ 为与入射电子束 方向垂直的 $(x, y)$ 平面内的位置矢量. 当电子束穿过 晶体样品时, 它的振幅和相位就会受到样品内部静 电势的影响, 经过一系列透镜成像后, 物波函数(穿
过样品的电子波)可表示为

$$
\phi(\boldsymbol{r})=A(\boldsymbol{r}) \cdot \mathrm{e}^{\mathrm{i} \varphi(\boldsymbol{r})},
$$

其中 $A(\boldsymbol{r})$ 为振幅, $\varphi(\boldsymbol{r})$ 为相位, 电子束穿过双棱镜 的干涉场之后, 记录下来的电子全息图是一个简单 的干涉调制图, 其强度可以表示为 ${ }^{[5]}$

$$
\begin{aligned}
I_{h}= & 1+A^{2}(\boldsymbol{r})+I_{\text {inel }}(\boldsymbol{r}) \\
& +2 \mu A(\boldsymbol{r}) \cdot \cos \left(2 \pi \boldsymbol{q}_{c} \boldsymbol{r}+\varphi(\boldsymbol{r})+\theta\right) .
\end{aligned}
$$

所得到的图是由物波函数和参考波函数经过 Möllenstedt-Düker 双棱镜干涉而成的, 其中 $I_{\text {inel }}(\boldsymbol{r})$ 为非弹性 散射强度项, $A(\boldsymbol{r})$ 为振幅, $\varphi(\boldsymbol{r})$ 为相位, 在 $A=1$, $I_{\text {inel }}$ 为常数的情况下, $\boldsymbol{q}_{c}$ 为电子全息图干涉条纹的空 间频率(有时称载波频率), 定义为 $2 \alpha / \lambda, \alpha$ 为双棱镜 的干涉半角, $\lambda$ 为入射电子的波长, $\mu$ 用来描述干涉条 纹的衬度. 所包含的振幅信息随着电子全息图干涉 条纹衬度的变化而变化, 而其中的相位会随着干涉 条纹的移动而变化. (3)式中前 3 项为记录下的电子全 息图的背底强度, 余弦函数项给出的是材料内部电 磁场与电子波相互作用后相位变化. 基本的重构过 程基于(3)式, 对其进行傅里叶变换就能提取所需要 的信息, 获得分离的振幅图和相位图.

$$
\begin{aligned}
\mathrm{FT}\left\{I_{h}(\boldsymbol{r})\right\}= & \delta(\boldsymbol{q})+\mathrm{FT}\left\{I_{\text {inel }}+A^{2}(\boldsymbol{r})\right\} \\
& +\delta\left(\boldsymbol{q}+\boldsymbol{q}_{c}\right) \otimes \mathrm{FT}\left\{A(\boldsymbol{r}) \cdot \mathrm{e}^{\mathrm{i}[\varphi(\boldsymbol{r})+\theta]}\right\} \\
& +\delta\left(\boldsymbol{q}-\boldsymbol{q}_{c}\right) \otimes \mathrm{FT}\left\{A(\boldsymbol{r}) \cdot \mathrm{e}^{-\mathrm{i}[\varphi(\boldsymbol{r})+\theta]}\right\} .
\end{aligned}
$$

傅里叶变换后的函数((4)式)是一个复函数, 分为三部 分, 第一项与背底有关, 对应于傅里叶变换的中心, 称为中心带. 来自 (3)式中余弦函数后面的两项被称 为边带 (上边带“upper sideband”, 下边带“lower sideband”), 有关电子全息图中振幅和相位的信息都来自 
边带, 这两个边带其中一个是另一个的共轭, 包含了 同样的信息. 图 $2^{[6]}$ 所示为 $\mathrm{GaAs}$ 晶体的电子全息图进 行傅里叶变换后中心带和边带的位置, 选择其中一 个边带移 $\pm q_{c}= \pm 2 \alpha / \lambda$ 到中心位置后, 进行反傅里叶 变换就可以获得振幅相位图.

$$
\begin{gathered}
\text { Phase }(\mathrm{obj})=\varphi(\boldsymbol{r})+\theta, \\
\operatorname{Mod}(\mathrm{obj})=A_{0} .
\end{gathered}
$$

把样品移出视场, 相同实验条件下重新记录没有样 品的参考波的电子全息图, 设定相位和振幅分别为

$$
\begin{aligned}
\text { Phase(ref) } & =0, \\
\operatorname{Mod}(\mathrm{ref}) & =A_{\mathrm{r}} .
\end{aligned}
$$

出射波相位相对于参考波的相位变化可以用(5)式表 示, $\mathrm{McCartney}{ }^{[7]}$ 指出样品的厚度可以用物波相对于 参考波的振幅 $A_{0} / A_{\mathrm{r}}$ 计算给出:

$$
\frac{t}{\lambda_{i}}=-2 \ln [\operatorname{Mod}(\mathrm{obj}) / \operatorname{Mod}(\mathrm{ref})]=-2 \ln \left(A_{0} / A_{\mathrm{r}}\right),
$$

$\lambda_{i}$ 为电子的非弹性平均自由程, 跟样品的成分和点 阵取向有关.

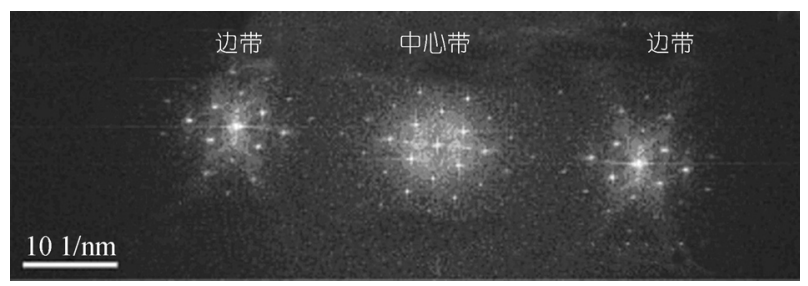

图 $2 \mathrm{GaAs}$ 的傅里叶变换谱 分别指出了两个边带和中心带的位置

每次记录参考波电子全息图，是可以比较精确 地确定傅里叶变换后边带真正的中心位置的原因, 从而给出一个合理的重构相位图. 另外利用参考波 还可以补偿由于入射光不均匀、双棱镜细丝的污染或 粗细不同等引起的图像变形.

\section{2 实验方法}

实验中影响电子全息最重要的因素是电子束的 相干性, 它直接影响电子全息图中干涉条纹衬度的 好坏. 我们采用的是椭圆光斑成像, 这种方法对任何 光源和聚光镜系统都适用, 人为地利用聚光镜的像 散和聚焦设置把光斑调节为椭圆, 使得在双棱镜细 丝的垂直方向很长, 而平行方向很窄. 也就是说椭圆 光斑的长轴方向和双棱镜丝垂直, 椭圆的短轴宽度 一般为物波和参考波干涉区域的 2 5 倍, 长轴的长度 约为干涉区域宽度的 50 100 倍, 如图 $3^{[8]}$ 所示. 另外 值得注意的是, 在调整聚光镜像散的时候, 一定要确
保椭圆长轴和双棱镜丝垂直, 否则相干性就会变差, 条纹衬度降低, 实际调节时在较低的双棱镜电压下 比较容易, 因为电压降低干涉条纹衬度提高, 而且条 纹间距变大.

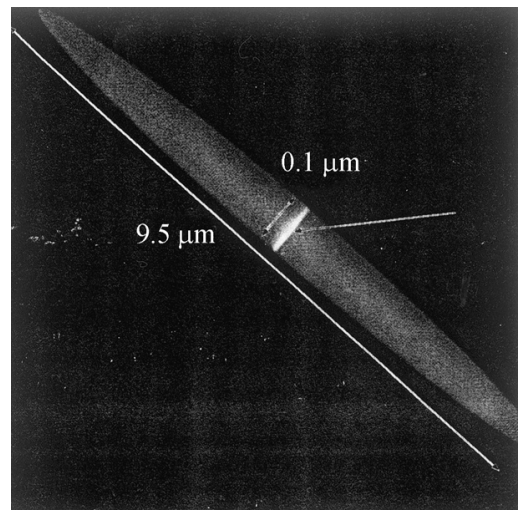

图 3 用来记录电子全息的光源情况

\section{3 主要参数}

条纹间距: 加在双棱镜上的电压越大意味着条 纹间距越小，观察范围(干涉区域)越大，但是条纹的 可见性就不可避免地降低. 分析表明, 条纹间距随双 棱镜电压和比例常数成反相变化, 比例常数依据仪 器参数不同而不同, 包括加速电压、物镜的焦长和物镜 背焦面与双棱镜之间的距离. 条纹间距和双棱镜电压 之间的关系可以计算出来. 图 $4^{[8]}(\mathrm{a})$ 是条纹间距随双棱 镜电压不同而变化的曲线(对于Philips CM200-FEG电 镜), 可以看出双棱镜电压在 150 240 V时条纹间距才 能达到 $1 \AA$. 要达到 $1 \AA$ 的空间分辨率，条纹间距应小 于 $0.33 \AA$, 加在双棱镜上的电压可能就需要到 500 700 V. 图 4(b)为干涉区域随双棱镜电压变化的曲 线(实线), 虚线为同样电压范围内条纹间距的变化. 一般情况下，根据这条曲线结合加在双棱镜上的电压， 可以确定所得到的干涉条纹间距和干涉区域的大小.

条纹可见性: 电子全息中干涉条纹的可见性对 重构过程非常重要, 它直接决定重构图像的相位精 度. 高压的稳定性、电子束的扩展、样品台的移动、 双棱镜的机械稳定性和电场稳定性都会影响条纹的 可见性. 条纹可见性有不同的评价方法 ${ }^{[9]}$, 标准的方 法是在没有样品的干涉图像中进行线扫描，利用关 系式 $\mu=\frac{I_{\text {max }}-I_{\text {min }}}{I_{\text {max }}+I_{\text {min }}}$ 表示. 另一种方法可以采用对全 息图进行傅里叶变换, 利用插补方法(interpolation method) ${ }^{10}$ 计算中心峰和两个边带的高度, 条纹的可 
见性定义为边带振幅的两倍与中心峰振幅的比值.
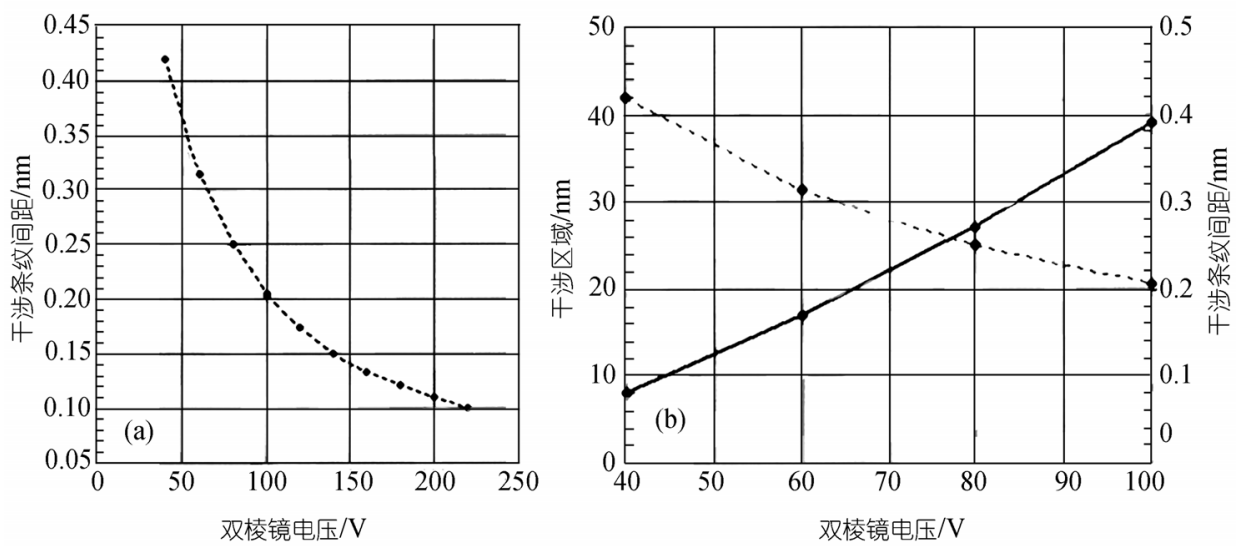

图 4 在 $200 \mathrm{kV}$ Philips CM200-FEG TEM 操作模式下, 测得的条纹间距(a)和干涉区域大小(b) 与双棱镜电压之间的关系

\section{2 电子全息术在材料中的应用}

除了电子全息技术本身的发展以及进行球差和 色差矫正 ${ }^{[11,12]}$ 应用外，电子全息在材料领域的各个 方面也获得了广泛应用，如在磁性材料中可以直接 观察磁畴、磁分布和磁转变等, 非磁性材料中样品的 平均内电势分布、晶体管和p-n结内电势、电荷密度 分布、缺陷结构及位错密度分布分析，铁电材料的极 化以及有关材料的三维形态的信息研究等.

电子波穿过样品时会受到材料内势场的调制, 如果受到样品静电势场吸引力的作用, 样品下表面 出射电子波的相位将比真空中沿同一方向的参考电 子波的相位超前. 如果受到静电排斥力的作用, 则出 射电子波的相位将落后于真空中参考电子波的相位. 入射电子波穿过磁性材料时会受到洛伦兹力的作用 而改变出射电子波的相位，通过对出射电子波相位 的测量可获得有关材料内部电磁场的信息. 高能电 子与电磁场的交互作用可用薛定谔方程表示:

$$
\left(\frac{1}{2 m} \cdot(-i h \nabla+e \boldsymbol{A})^{2}-e V\right) \phi=E \phi ，
$$

其中 $m, e, h, \phi, A, V$ 和 $E$ 分别为电子质量、电荷绝对 值、普朗克常量、波函数、矢量势、标量势和电子能 量. 只考虑弹性散射, 物波函数如(2)式所示, 根据 WKB 假设解出 (10)式为

$$
\phi(x, y)=\frac{\pi}{\lambda E} \cdot \int_{L} V(x, y, z) \mathrm{d} z-\frac{2 \pi e}{h} \cdot \int_{L} A z(x, y, z) \mathrm{d} z .
$$

积分沿平行于光轴方向的直线 $L$ 进行, 从(11)式可以 看出，相位依赖于电势而不是电场.
对于非磁性材料, 我们假设矢量势为零, 则(11) 式变为

$$
\phi(x, y)=\frac{\pi}{\lambda E} \cdot \int_{L} V(x, y, z) \mathrm{d} z .
$$

设定样品的平均内电势为 $V_{0}$, 厚度为 $t$, 材料内电势 $V(x$, $y, z$ 为常数, $z$ 为电子束的入射方向, $x$ 和 $y$ 为入射电子束 垂直方向. 如果不考虑沿电子束方向电势 $V$ 和样品厚 度 $t$ 的不均匀变化, 那么沿电子束入射方向对电势进 行积分，则得到相位和电势之间的简化关系式，可以 表示为 $\phi(x, y)=(\pi / \lambda E) V_{0} t$, 其中 $E=\left(U_{A} / 2\right)\left(2 m_{0} c^{2}+\right.$ $\left.e U_{A}\right) /\left(m_{0} c^{2}+e U_{A}\right)$, 可以得到

$$
\phi=\frac{2 \pi}{\lambda U_{A}} \cdot \frac{2 m_{0} c+e U_{A}}{m_{0} c^{2}+e U_{A}} \cdot V_{0} \cdot t=C_{E} V_{0} t,
$$

其中 $U_{A}$ 为电子的加速电压, $C_{E}$ 是与样品无关的电子 显微镜参数, 对于加速电压为 $200 \mathrm{kV}$ 的电子显微镜 $C_{E}=7.295 \times 10^{-3} \mathrm{rad} /(\mathrm{V} \cdot \mathrm{nm})$, 对于加速电压为 $300 \mathrm{kV}$ 的电子显微镜 $C_{E}=6.25 \times 10^{-3} \mathrm{rad} /(\mathrm{V} \cdot \mathrm{nm})$. 根据(13)式, 如果已知样品厚度就可以计算出材料的平均内电势, 同样如果材料内电势已知就可以给出样品的厚度 [13 16]. 图 5给出了氧化锆纳米颗粒的高分辨像和重构 出的相位图，以及不同方向不同位置的相位分布曲 线，根据给出的相位图我们可以确定颗粒的三维立 体形貌 ${ }^{[17]}$, 而这是从透射高分辨像中无法得到的.

\section{1 界面附近的电势分布}

电子全息最早用于磁性材料领域中研究铁磁薄膜 的磁畴, 在高密度磁记录介质的发展中起到了重要的 作用 $[18-21]$, 在磁性材料研究经验基础上，Frabboni等人 
[22]首先把电子全息作为一门测量技术，给出了第一

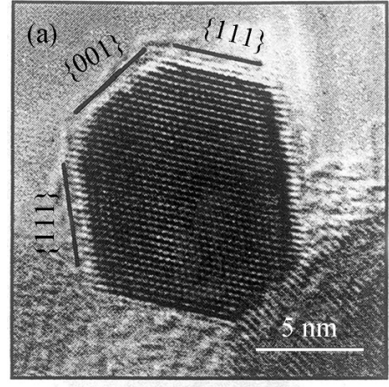

(b)
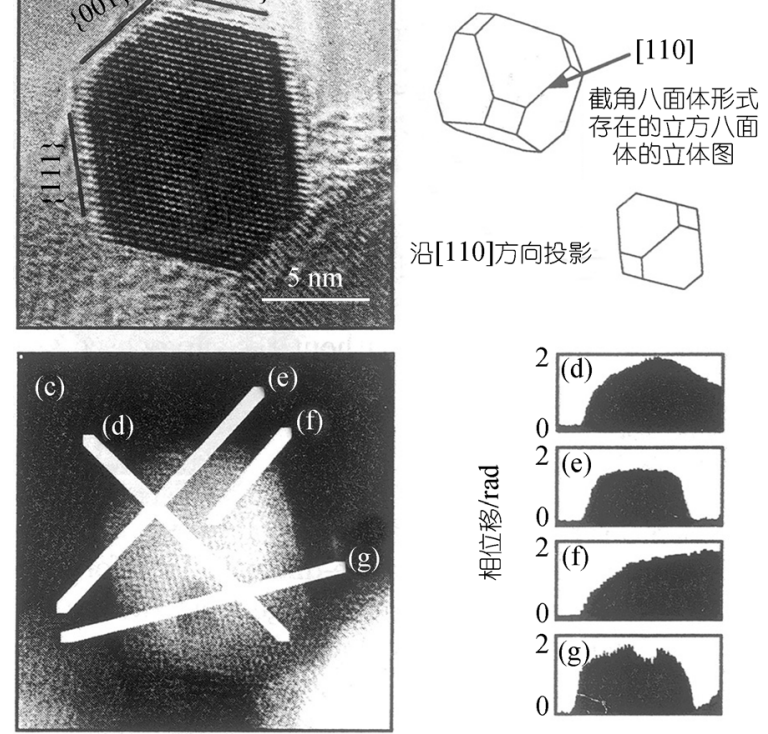

图 5 氧化锆纳米颗粒的结构及电子全息结果

(a) 氧化铅颗粒的高分辨像; (b) 相应的立方八面体结构和 [110]方向 投影; (c) 从(a)位置记录的电子全息图中重构出的相位图; (d), (e), (f) 和 $(\mathrm{g})$ 分别为 $(\mathrm{c})$ 中所示的沿不同方向的相位变化曲线. 实验结果与设 想一致, 颗粒中存在空位如 $(\mathrm{g})$ 所示

张硅基p-n结周围电势分布图. 因为界面对材料的性能 有非常重要的影响, 到目前为止, 有关电子全息在材料 界面问题的研究已有相当一部分有意义的工作 ${ }^{[2329]}$, 包括钛酸锶双晶界面、异质结(同型异质结和异型异质 结)界面势垒高度和宽度、电场、电荷密度及其分布.

(i ) 无定形和多晶异质界面 Weiss等人 先采用电子全息研究了 $\mathrm{Mo} / \mathrm{Si}$ 多层结构, 依据线性 和非线性拟合方法排除厚度引起的误差得出相位分 布, 从重构出的振幅图定出 $\mathrm{Mo} / \mathrm{Si}$ 体系中每层成分, 从相位图中推出 $\mathrm{Si}$ 的平均内电势比 Mo的低, 大致估 计了 $\mathrm{Si}_{3} \mathrm{~N}_{4}$ 陶瓷复合材料中界面的非晶层厚度. 图 6 为Weiss给出的第一张相位图, 为无定形 $\mathrm{Si}$ 和多晶 $\mathrm{Mo}$ 交错生长结构, 线扫描结果表明除了相位和厚度有 关外, Si的相位比Mo的相位高, 同时也表明电子全息 对界面的敏感性.

(ii ) 半导体硅基异质结 对于半导体装置( $p-n$, $p-n-p, n-p-n)$ 来说, 静电势分布与掺杂原子分布有关. 穿 过半导体异质结的相位变化可以简单表示为 $\phi(x, y)=$ $C_{E} V(x, y) t(x, y)$, 其中静电势 $V(x, y)$ 主要包括三部分: 一部分是样品的平均内电势 $V_{0}$ 信息, 它只与材料的 成分、密度和原子种类有关，不同材料的平均内电势 有所不同，大小一般在 $-30 \sim-5 \mathrm{~V}$. 表 1 给出了一些采
用电子全息测出的材料平均内电势的信息. 测量比
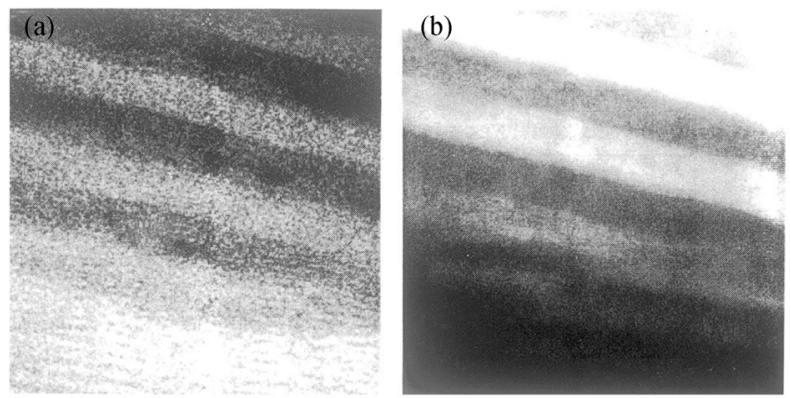

图 6 采用电子全息研究异质界面获得的振幅(a)和相位(b)

表 1 利用电子全息技术测出的不同材料的平均内电势

\begin{tabular}{|c|c|c|}
\hline 材料 & $V_{0} / \mathrm{V}$ & 文献 \\
\hline \multirow{6}{*}{$\mathrm{Si}$} & $11.9 \pm 0.7$ & $\mathrm{Rau}$ 等[29] \\
\hline & $12.0 \pm 0.2$ & Li等 $[14]$ \\
\hline & $9.26 \pm 0.08$ & Gajdardziska-Josifovska等 ${ }^{[13]}$ \\
\hline & $12.1 \pm 1.3$ & Wang等 $[30]$ \\
\hline & $12.16 \pm 0.83$ & Wang等[31] \\
\hline & $11.5 \pm 0.5$ & $\mathrm{Wu}$ 等 $[32]$ \\
\hline Si(无定形) & $11.9 \pm 0.9$ & \multirow{2}{*}{ Wang等 [30] } \\
\hline $\mathrm{SiO}_{2}$ (无定形) & $10.1 \pm 0.6$ & \\
\hline $\mathrm{MgB}_{2}$ & $11.7 \pm 0.9$ & $\mathrm{Wu}$ 等 $[32]$ \\
\hline \multirow[t]{2}{*}{$\mathrm{ZnO}$} & $\begin{array}{c}21 \pm 4.2 \text { (实验) } \\
16.1 \text { (理论) }\end{array}$ & Elfwing等 [33] \\
\hline & $15.9 \pm 1.5$ & Müller等 $[34]$ \\
\hline $\mathrm{MgO}$ & $\begin{array}{c}13.01 \pm 0.08 \\
14\end{array}$ & $\begin{array}{c}\text { Gajdardziska-Josifovska等 } \\
\text { Yada等年 }\end{array}$ \\
\hline $\mathrm{PbS}$ & $17.19 \pm 0.17$ & Gajdardziska-Josifovska等 ${ }^{[13]}$ \\
\hline $\mathrm{GaN}$ & $11 \pm 3$ & Cherns等 ${ }^{[36]}$ \\
\hline Gan & 13.5 & McCartney等 ${ }^{[37]}$ \\
\hline GaAs & $\begin{array}{c}14.53 \pm 0.17 \\
14.18\end{array}$ & Gajdardziska-Josifovska等 ${ }^{[13]}$ \\
\hline $\begin{array}{l}\text { InAs } \\
\text { GaP } \\
\text { InP }\end{array}$ & $\begin{array}{l}14.50 \\
14.35 \\
14.50\end{array}$ & Kruse等 $[15]$ \\
\hline $\mathrm{Ge}$ & $14.3(2)$ & $\mathrm{Li}$ 等 $[14]$ \\
\hline $\mathrm{Au}$ & $\begin{array}{c}21 \sim 23(>5 \mathrm{~nm}) \\
>30(<5 \mathrm{~nm}) \\
>40(<2 \mathrm{~nm})\end{array}$ & \multirow[t]{2}{*}{ Ichikawa等 [38] } \\
\hline $\mathrm{Pt}$ & 约 $25(>1.5 \mathrm{~nm})$ & \\
\hline $\mathrm{Cu}$ & 21.2 & Wang等 $[39]$ \\
\hline 碳纳米管 & $\begin{array}{l}24 \pm 4 \text { (雉型) } \\
20 \pm 6 \text { (单层) }\end{array}$ & Lin等 $[40]$ \\
\hline
\end{tabular}

较多的是 $\mathrm{Si}$ 的内电势, 不同研究小组测出的值有微小 差异, 但基本和理论值是一致的; 另外一部分, 由于 掺杂浓度和掺杂原子的变化引起空间电荷势或掺杂 势, 也就是内电场. 关于内电场, 研究人员做了很多 研究, 如陶瓷晶粒界面电荷, 氮化物中的极化场分布 以及硅基电子材料内部掺杂原子分布等; 还有就是 样品表面的静电场也会引起电势的变化, 对于导电 样品, 如果样品表面集聚净电荷很少, 这个外电场对 
电势的贡献一般很小, 可以忽略; 对于不导电样品, 样品表面可能聚集高密度的电荷, 样品表面要喷涂 一层导电保护层以确保不会由于样品表面聚集电荷 引起外电场影响. 因此在忽略外电场的情况下, 电子 束穿过样品之后引起的相位变化可以表示为 $\phi(x, y)=$ $C_{E}\left[V_{0}+V_{\mathrm{pn}}(x, y)\right] t(x, y)$, 有关外电场和样品表面电荷 对电子波相位的影响可参考文献[41,42].

图 7 ${ }^{[43]}$ 给出了 $\mathrm{p}-\mathrm{n}$ 异质结结构示意图以及耗尽层 附近的电势、电场和电荷密度分布图. 电子全息可以 用一维或者二维图像很直观地描述出异质结中电势 分布, 但要定量地精确分析晶体管结构, 必须考虑到 一些实际问题, 如 Rau指出, 典型的 $\mathrm{Si}$ 半导体由于表 面的耗尽效应, 截面样品制备过程中上下表面会有 一层约 $25 \mathrm{~nm}$ 厚的非晶死层 (dead layer $)^{[29]}$, 设其厚度 为 $t_{0}$, 样品的总厚度为 $t$, 那么电子束穿过 $\mathrm{p}-\mathrm{n}$ 结后相 位变化可以写为

$$
\phi=C_{E}\left[V_{0} t+V_{\mathrm{pn}}\left(t-2 t_{0}\right)\right] .
$$

要测出穿过异质结的相位变化值, 需要知道样品的平 均内电势和样品的厚度, 样品表面死层的厚度. $\mathrm{Rau}^{[29]}$, Gajdardziska-Josifovska ${ }^{[13]}$, McCartney ${ }^{[44]}$ 和Wang ${ }^{[45]}$ 等人 采用聚焦离子束减薄的方法制备已知角度的楔形样品, 采用电子全息测出穿过样品的相位变化, 得到了内电 势大小, 在楔形角已知的情况下就可以知道样品的 厚度, 扣除平均内电势和样品厚度的贡献, 根据 $V_{\mathrm{pn}}=\frac{\phi(x, y)}{C_{E} t(x, y)}-V_{0}$ 就可以计算出界面势垒的分布. 样 品的厚度还可以利用(9)式提到的重构出的振幅图和 (a)
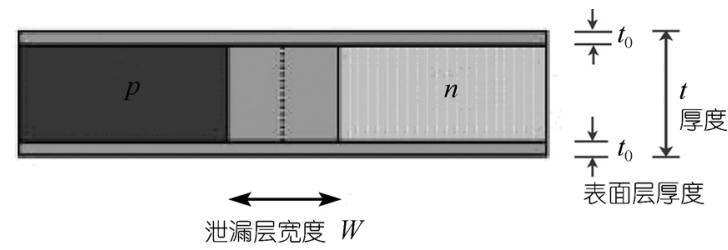

(b)

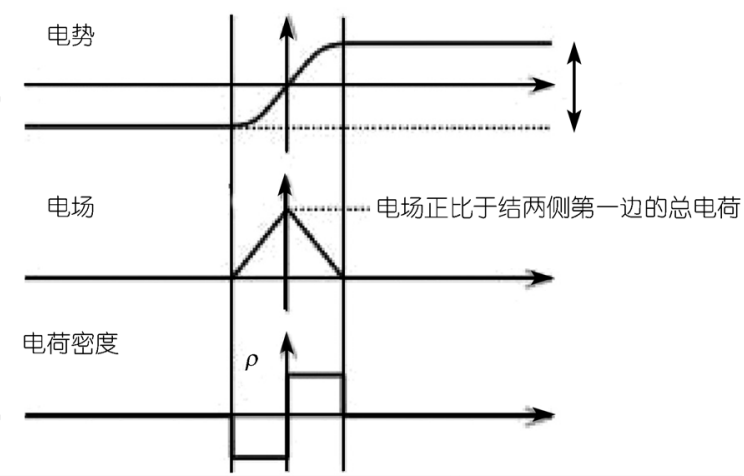

图 7 p-n 异质结结构示意图(a), 以及耗尽层附近的电势 (b)、电场 $(\mathrm{c})$ 和电荷密度 $(\mathrm{d})$ 分布示意图 材料的平均自由程得出, 即 $t / \lambda_{i}=-2 \ln \left(A_{0} / A_{\mathrm{r}}\right)$, 或者 在样品厚度未知的情况下, 根据平均自由程求出样品

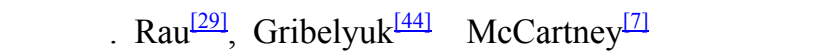
了CMOS晶体管中内电势的分布情况, 图 $8^{[46]}$ 是场效 应管的低倍明场形貌像和重构出的相位分布图. 可 以看出, 从普通的TEM图中看不到细节性的问题, 而 从相位图中可以很清楚地看到晶体管的源区、漏区、 门和侧壁隔离物各个部分的分布情况. 在半导体工业 中, 随着半导体尺寸的逐渐降低, 研究掺杂原子的分 布情况将会有非常重要的作用.

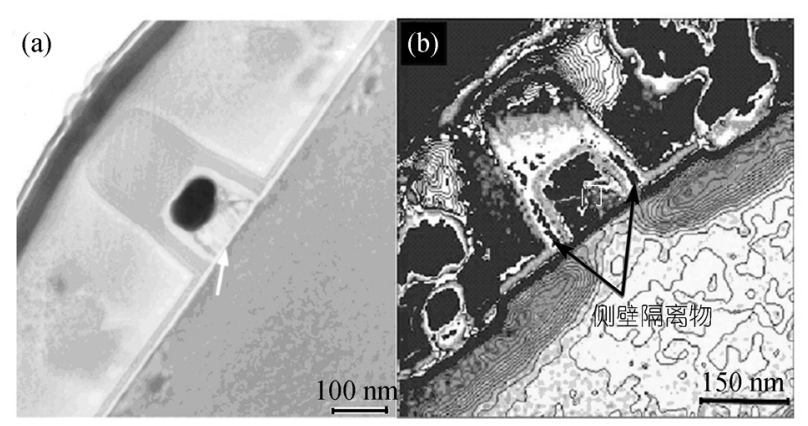

图 $80.13 \mu \mathrm{m}$ 场效应管的 TEM 图(a)和从记录的电子 全息图中重构出的相位分布(b)

Twitchett 等人 ${ }^{[3,43,47,48]}$ 设计了一种特殊的样品 台, 研究了 $\mathrm{Si}$ 掺杂的 $\mathrm{p}-\mathrm{n}$ 结系统. 样品采用FIB方法 制备, 且样品上可加正反偏压, 由于平均内电势为一 常数, 对相位的影响只是在整体上增加一个常数, 因 此可以忽略不计. 利用这种方法, 根据电子全息可以 直接得到样品的厚度、死层厚度、内电势大小以及耗 尽层的宽度.

图 9 给出了电子全息结果, 其中图 9(a)和(b)为 p-n 结相位随偏压和样品厚度的变化曲线, 图 9(c)和 (d) 是从 (a)和(b)中得出的相位大小与偏压和样品厚度 的变化关系. 可以看出, 图 $9(\mathrm{c})$ 曲线的斜率就是 $C_{E}\left(t-t_{\text {dead }}\right)$, 从而就可以得到样品表面非晶层的厚 度; 而曲线在垂直坐标轴的截距就是 $C_{E} V_{\mathrm{bi}}\left(t-t_{\mathrm{dead}}\right)$, 根据已知的数据就可以得出异质结的内电势. 图 9(d) 中曲线偏离直线, 表示样品表面非晶层的厚度随晶 体样品厚度的减小而增加. 图 9(e)和(f)给出了耗尽层 的宽度随所加偏压和晶体样品厚度变化的大致曲线.

根据 $\mathrm{p}-\mathrm{n}$ 结中电势分布, 电场 $E(x)=-d_{V} / d_{x}$ 和 电荷密度 $\rho(x)$ 之间的关系可以利用泊松方程得出: 
$\rho(x)=-\left(1 / \varepsilon \varepsilon_{0}\right) \frac{\partial^{2} V(x)}{\partial x^{2}}$, 式中 $\varepsilon$ 和 $\varepsilon_{0}$ 分别是样品和真
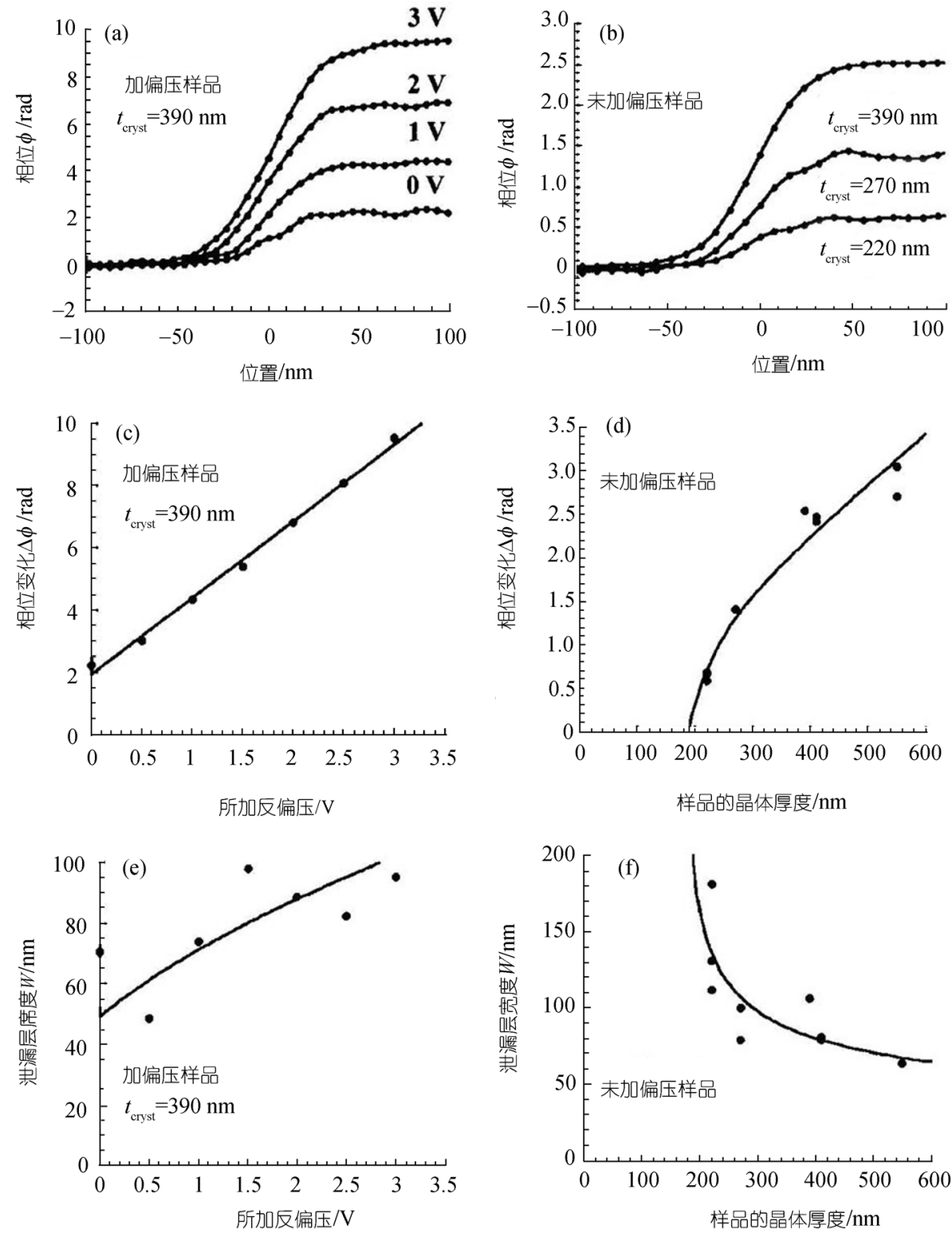

图 9 p-n 结相位及耗尽层宽度随偏压和样品厚度的变化关系

(a), (b) p-n 结相位随偏压和样品厚度的变化曲线; (c), (d) 从(a)和(b)中得到的相位大小与偏压和样品晶体厚度的变化关系;

(e), (f) 耗尽层的宽度随所加偏压和晶体样品厚度的变化曲线

空的介电常数, 电势、电场和电荷密度三者之间关系 如示意图 8 所示.

(iii) $I I I-V$ 族半导体量子阱界面 $\mathrm{GaN}$ 材料作为 器件已经在场发射二极管、激光二极管、紫外探测器 以及微波、高温、高压转换器中获得了广泛应用. Cherns ${ }^{[49]}$ 和 $\mathrm{Jiao}^{[50]}$ 等人采用电子全息对 $\mathrm{n}$ 型 $\mathrm{GaN}$ 中位 错所带电荷的类型和位错密度进行了研究, 给出了
非常漂亮的结果. 图 10 是 $\mathrm{GaN}$ 材料内部位错周围的 相位分布图以及穿过位错中心的电势分布曲线的实 验和理论值比较. GaN之所以有很强的发光特性与其 内部存在高密度的位错有关, 电子全息结果表明位 错带负电荷, 电荷密度为 $4 \times 10^{7} \mathrm{e} / \mathrm{cm}$, 且实验与理论 吻合相当好.

Cherns $\left.{ }^{3} 6\right\}$ 和 $\mathrm{Cai}^{5151,52]}$ 等人首先采用电子全息研究 
了 $\mathrm{GaN} / \mathrm{In}_{x} \mathrm{Ga}_{1-x} \mathrm{~N} / \mathrm{GaN}$ 量子阱中的电势和电荷分布,

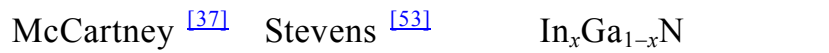
构中由于极化产生的内电场分布进行了研究, 忽略 量子阱厚度不均匀对静电势的影响, 认为量子阱界
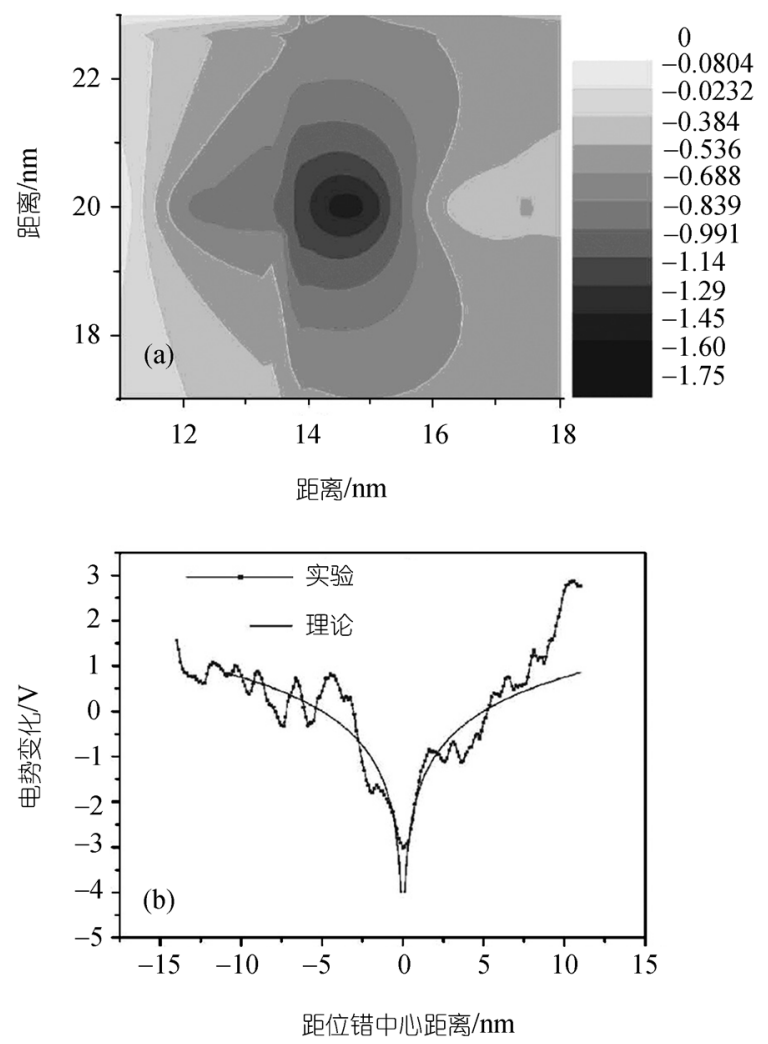

图 10 位错中心附近的相位及电势变化曲线

(a) $\mathrm{GaN}$ 材料内部位错中心附近的相位图, 表明相位逐渐降低;

(b) 沿相位图中水平方向穿过位错中心的电势变化曲线, 实验与理论吻合

面净电荷分布主要是由界面自发极化、压电极化、载 流子聚集和界面偶极距产生的.

(iv) 钙钛矿氧化物异质结(钛酸锶双晶异质结, $\mathrm{La}(\mathrm{Sr}) \mathrm{MnO}_{3} / \mathrm{Sr}(\mathrm{Nb}) \mathrm{TiO}_{3}$ 异质结) Ravikumar等人 ${ }^{[23]}$ 首先提出用电子全息来研究钛酸锶电子陶瓷双晶异 质界面，由于界面两侧晶体的斜切角度相同，因此界 面两侧晶体的厚度、平均内电势和动力学衍射效应的 贡献都可以看作是相同的，这就解决了界面两侧材
料因平均内电势的不同而导致的一些误差和动力学 效应, 因此在界面处得到的相位变化可以看作是界 面处空间电荷的分布. 由于掺铌钛酸锶双晶界面 $I-V$ 曲线的非线性, Johnson等人 ${ }^{[24]}$ 采用原位加电流的方 法研究掺铌钛酸锶双晶界面势垒的衰减过程, 在加 上电流以后, 界面势垒明显被抑制, 从原来 $0.45 \mathrm{~V}$ 的 界面势垒高度降为很小, 几乎跟噪音分不出来, 可以近 似为零, 未加电流和加电流之后的相位有了明显变化.

我们采用电子全息对 $\mathrm{LaAlO}_{3}$ 基体上生长的 $\mathrm{Ba}_{0.5}$ $\mathrm{Sr}_{0.5} \mathrm{TiO}_{3}(\mathrm{BST})$ 薄膜进行了表界面研究 ${ }^{[54]}, \mathrm{BST}$ 薄膜在 $250 \mathrm{~K}$ 有铁电转变. 图 11 是BST薄膜在温度为 $120 \mathrm{~K}$ 时得 到的电子全息图和从一系列电子全息图中重构出的相位 变化图, 以及BST薄膜表面的相位变化曲线和扣除薄膜 内电势和样品厚度变化的因素得到的薄膜表面的电势变 化曲线. 图 12 为界面的全息图和相应的相位分布、电势 分布变化曲线, $120 \mathrm{~K}$ 的电子全息结果表明, 由于铁电极 化导致界面有明显的正电势, 表面有负电势存在. 图 $12(\mathrm{~d})$ 给出了原子运动模型, 由于 $\mathrm{Ba}(\mathrm{Sr})$ 和 $\mathrm{O}$ 原子的相 对运动导致界面有正电荷聚集，表面有负电荷聚集. 同 时我们在室温下对样品的缺陷进行了研究, 如图 13 所 示, 结果表明界面位错处和表面台阶等缺陷处都有电荷 的聚集, 致使介电函数曲线呈现蝶形(图 13 (a)).

我们还对具有钙钛矿结构的锰基异质结进行了研 究 ${ }^{[55]}$. 这种异质结是近年来材料研究的热点, 这种新 半导体型异质结除了具有普通半导体的整流特性外, 还具有普通半导体异质结所不具有的独特的物理性 能, 如巨磁阻(正负磁阻)和电子相分离等, 与其他钙 钛矿结合起来制备的异质结具有很好的调谐性, 因 此对这种异质结界面势垒和电荷扩散的理解，无论对 科学研究还是技术应用都有很重要的意义. 图 14(a)为 质量分数为 $0.01 \% \mathrm{Nb}$ 掺杂 $\mathrm{SrTiO}_{3}(\mathrm{SNTO} 1)$ 基体上生长 

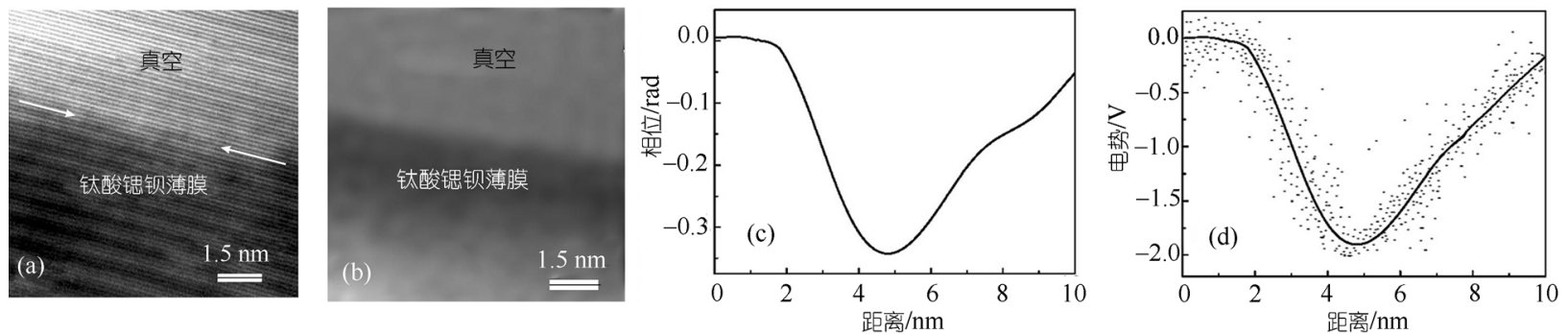

图 11 BST 薄膜表面电子全息结果

(a) $120 \mathrm{~K}$ 时 BST 薄膜表面的电子全息图; (b) 从全息图中重构出的相位图; (c) BST 薄膜表面相位变化曲线, 表明薄膜表面相位低; (d) 从薄膜表面到 真空的电势变化曲线, 会聚束电子衍射确定样品的厚度
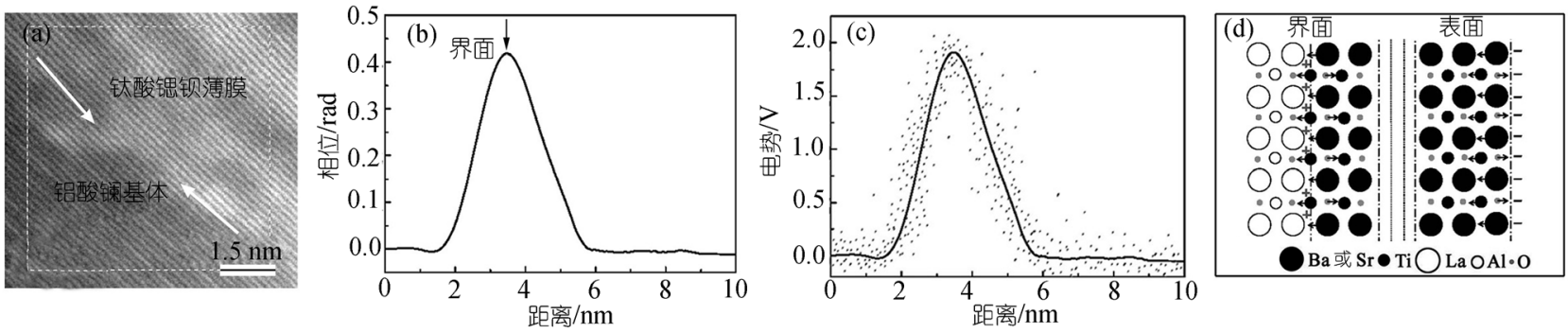

图 $12 \mathrm{BST} / \mathrm{LAO}$ 界面电子全息及原子相对运动示意图

(a) $120 \mathrm{~K}$ 时界面的电子全息图; (b) 穿过 BST/LAO 界面的相位变化曲线; (c) 界面的电势分布; (d) 薄膜内部由于自发极化引起的原子的相对运动示意图
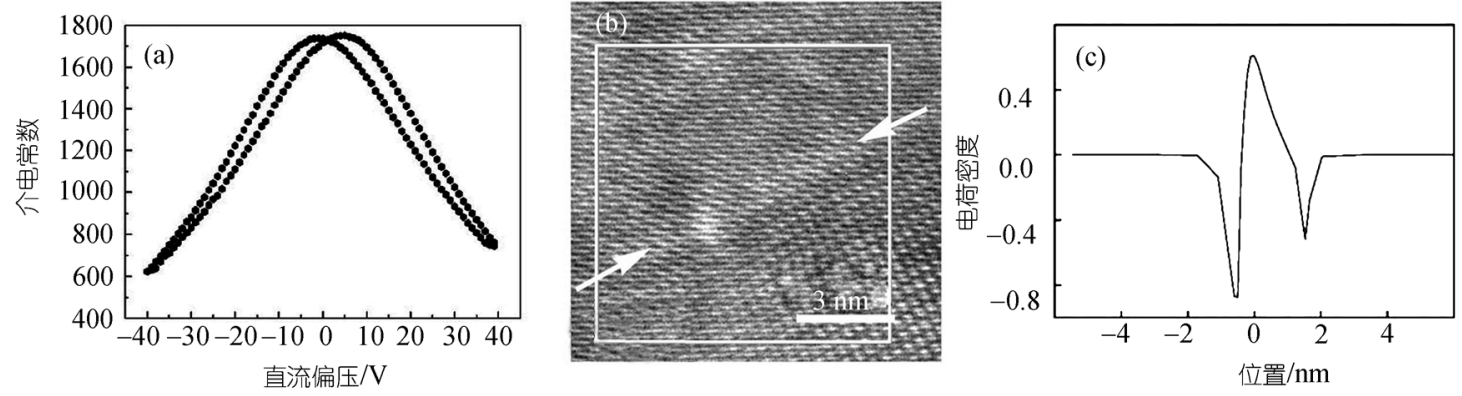

图 $13 \mathrm{BST} / \mathrm{LAO}$ 介电常数及界面单个位错的电子全息结果

(a) $300 \mathrm{~K}$ 时 $1 \mathrm{MHz}$ 下介电常数随直流偏压的变化关系; (b) $300 \mathrm{~K}$ 时界面的电子全息图，有个明显的位错存在; (c) 位错附近的电荷分布
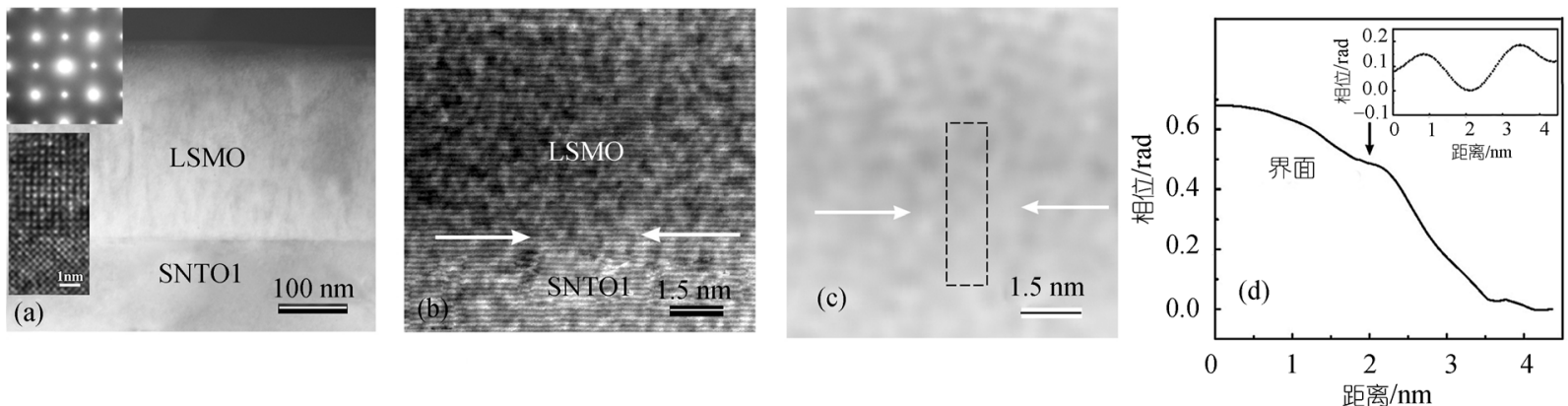

图 $14 \mathrm{LSMO} / \mathrm{SNTO} 1$ 薄膜的形貌及电子全息结果

(a) 质量分数 $0.01 \% \mathrm{Nb}$ 掺杂 $\mathrm{SrTiO}_{3}$ ( $(\mathrm{SNTO})$ ) 基体上 $\mathrm{La}_{0,9} \mathrm{Sr}_{0.1} \mathrm{MnO}_{3}$ (LSMO)薄膜的形貌像, 插图为界面电子衍射和高分辨像; (b) $300 \mathrm{~K}$ 时的电 子全息图, 箭头给出界面; (c) 重构出的相位图; (d) 穿过 p-n 结的相位变化曲线, 线扫描区域如(c)所示 
的 $\mathrm{La}_{0.9} \mathrm{Sr}_{0.1} \mathrm{MnO}_{3}$ (LSMO)薄膜的形貌像, 图 14(b), (c) 和 (d)分别为界面的电子全息图、重构出的相位图和相位 图上进行一定宽度的线扫描(图 14(c)中矩形)得到的穿 过 p- $n$ 结的相位变化曲线. 可以看到界面处有个小的结 (kink), 这与理论是基本一致的，因为薄膜和基体之间 介电常数的不同会导致界面电场的不连续性和电荷分 布的复杂性. 扣除样品厚度和内电势的影响之后, 我们 把穿过 $p-n$ 结的电势分布曲线表示为两个电势函数, 对 其进行求导可以得到界面附近的电场和电荷分布. 除
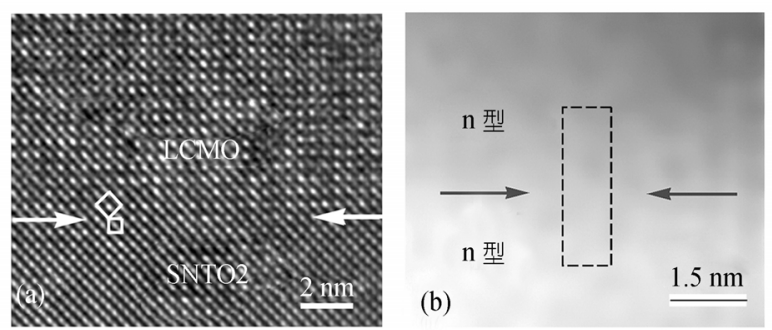

了对这种 p-n 结进行研究外, 我们还对 $\mathrm{La}_{0.7} \mathrm{Ce}_{0.3} \mathrm{MnO}_{3}$ (LCMO)/质量分数 $0.5 \% \mathrm{Nb}$ 掺杂 $\mathrm{SrTiO} 3$ (SNTO2) 同 型异质结(n-n) 结进行了系统分析. 图 15 给出了这种 异质结的高分辨像、重构出的相位图、电势分布图和 根据泊松方程得出的电荷密度分布. 从相位图上可以 明显地看出从基体到薄膜的相位变化, 亮的区域表示 相位高一些, 可以看出这种 $n-n$ 结也具有 $p-n$ 结的特性, 由于介电常数和价带宽度不同也会形成界面势垒层, 界面会有明显的电荷重新分布现象. 对其进行低温 120
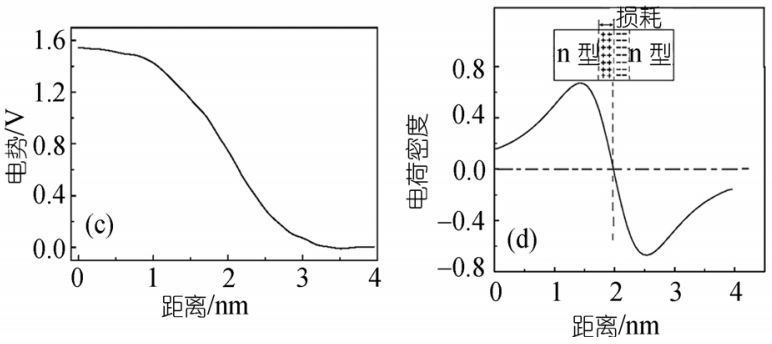

图 $15 \mathrm{LCMO} / \mathrm{SNTO} 2$ 薄膜的高分辨率像及电子全息结果

(a) 质量分数 $0.5 \% \mathrm{Nb}$ 掺杂 $\mathrm{SrTiO}_{3}$ 基体上 $\mathrm{La}_{0.7} \mathrm{Ce}_{0.3} \mathrm{MnO}_{3}$ (LCMO)薄膜的高分辨像; (b) 重构出的相位图; (c) $300 \mathrm{~K}$ 时穿过 $\mathrm{n}-\mathrm{n}$ 的电势变化; (d) $300 \mathrm{~K}$ 时穿过 $\mathrm{n}-\mathrm{n}$ 的电荷分布, 插图为结两侧空间电荷分布示意图

$\mathrm{K}$ 的电子全息研究, 结果表明低温下界面势垒层的高 度和势垒宽度几乎是室温时的两倍, 这与电性能的测 量结果是一致的. 图 16 为电性能测量结果和电子全息 测量结果的比较.

（v）隧道结势场分布 在金属层中插入氧化物 时, 由于内势场的差异会在界面形成阻挡电子穿过 界面的势垒. 这种界面势垒可以用来形成界面反射 巨磁电阻自旋阀或隧道结, 提高磁电阻率. 由电子全 息图得到的势场分布可以表征隧道结中金属/氧化物 界面特征, 与物理性能联系起来. 高分辨电子显微像 只能揭示界面的几何形态, 如界面的平整度以及位 错分布等, 特别是氧化物层为非晶态结构时, 界面的 失配位错消失, 难以将显微结构和物理性能联系起 来. $\mathrm{Xu}^{[56]}$ 和 $\mathrm{Shen}^{[57]}$ 等人研究了 $\mathrm{CoFe} / \mathrm{AlO}_{x} / \mathrm{Co}$ 磁性隧 道结 $(\mathrm{MTJ}$ ) 随退火温度变化的内电势变化. 制备态 的氧化层是阻碍电子输运的势垒, 退火处理后氧化 层变成一个束缚电子的势阱. 这是由于退火处理过程 中金属元素或元素团扩散进入氧化层, 使得一些电子 被束缚在氧化层内, 形成的静电场对通过氧化层的电 子波起到排斥作用，使出射电子波的相位滞后，从而使
被束缚在氧化层内, 形成的静电场对通过氧化层的电 子波起到排斥作用, 使出射电子波的相位滞后, 从而使 隧道结中电势随退火温度变化, 由势垒转变成势阱.

\section{2 磁性材料中的磁畴分布}

电子全息在磁性材料研究方面也有很大的发展, 主要有磁性颗粒内部磁畴分布、纳米线中磁力线和电

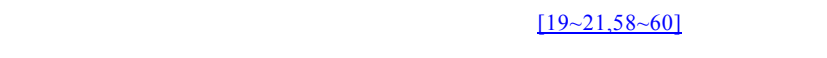
中磁通量观察 ${ }^{[61 ~ 65]}$, 尤其是对磁畴 ${ }^{[66 ~ 71]}$ 的研究获得 了突飞猛进的发展. 对于采用电子全息研究磁性材 料理论不详细介绍, 详见参考文献[72].

磁性材料的研究比较复杂, 根据(11)式, 电子波 相位除了对磁场分布敏感外, 同样也受样品内电场 分布影响, 因此要研究材料内部磁场分布, 需要把磁 场和电场对相位的贡献区分开来. 区分磁场和电场 对相位的贡献有几种方法，一种就是记录两个全息 图, 一个相位变化是 $\phi_{O 1}=\phi_{e}+\phi_{m}$, 同样状态下把样 品反转记录下的全息图的相位为 $\phi_{O 2}=\phi_{e}-\phi_{m}$, 两式 相加或相减就可以把电磁场分开. 还可以利用同一 个样品在不同加速电压下收集两张全息图, 由于磁 

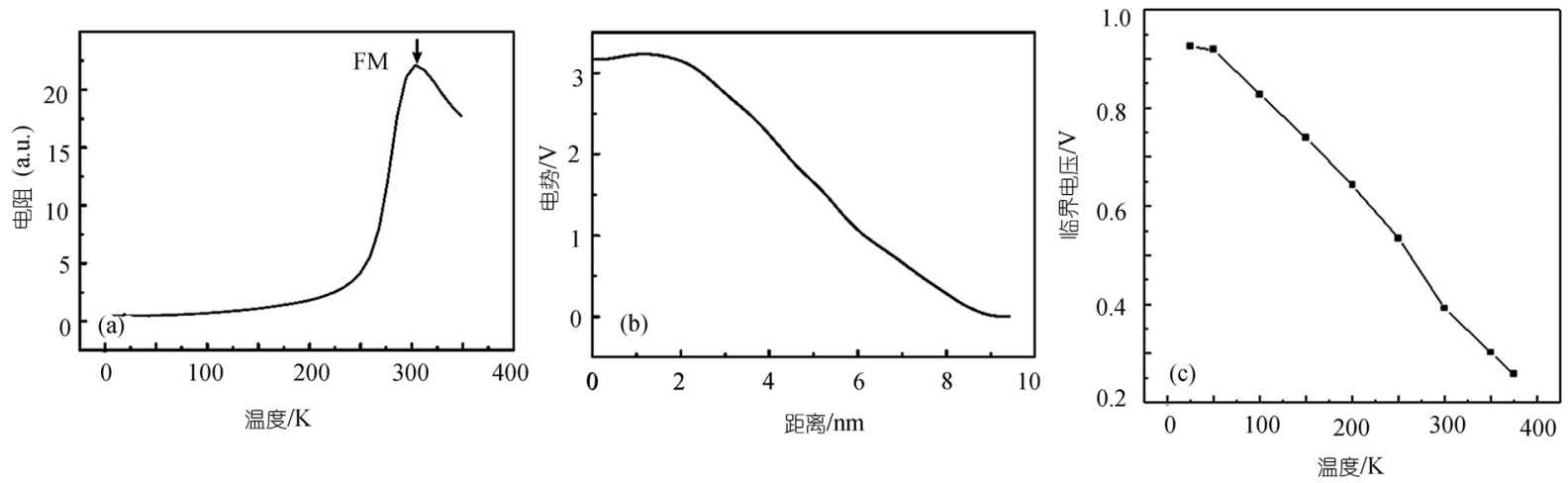

图 $16 \mathrm{La}_{0.7} \mathrm{Ce}_{0.3} \mathrm{MnO}_{3}$ 薄膜的电性能及电子全息测量结果

$\mathrm{La}_{0.7} \mathrm{Ce}_{0.3} \mathrm{MnO}_{3}$ (LCMO) 薄膜电阻随温度变化曲线, $300 \mathrm{~K}$ 时有个铁磁转变; (b) $120 \mathrm{~K}$ 时 $\mathrm{n}-\mathrm{n}$ 结电势变化曲线, 与室温电势相比有明显的提高; (c) 临界电压随温度变化曲线

场和加速电压无关，就可以得到电场的信息. Tonomura等人 ${ }^{[73]}$ 成功地采用电子全息把电场和磁场区分 开来, 描述了 $\mathrm{Co}$ 纳米颗粒中的磁力线分布. 由于电 子束穿过样品会受到材料内势场的调制, 如果受到 吸引力的作用, 样品下表面出射电子波的相位将比 真空中沿同一方向传播的参考电子波的相位超前; 如果受到排斥力的作用, 则出射电子波的相位将落 后于真空中参考电子波的相位. 入射电子波穿过磁 性材料时, 波阵面提前或者滞后与磁力线的旋转方 向是顺时针还是逆时针有关, 干涉图中颗粒中心突 出呈“山”形, 表明电子波穿过Co纳米颗粒中心后出 射电子波阵面超前, 因此可以确定磁力线方向是顺 时针的(图 17 (c)), 而图 17(a)和(b)中普通的显微镜形 貌和同轴电子全息都不能确定磁力线的方向.

近年来, 锰氧化物由于一些独特的物理性质, 引起了科学家们的广泛关注, 如巨磁阻、磁场诱导结 构相变、锰离子的电荷有序化, 以及降温过程中的金 属-绝缘体相变研究, 还有一些磁记录介质的应用, 尤 其是对磁畴的观察可以用来解释其一些独特的效应, 如铁磁畴和电荷有序相共存可以用来解释这种氧化物 的巨磁阻效应. Murakami和Yoo等人采用电子全息系统 地研究了 $\mathrm{La}_{0.46} \mathrm{Sr}_{0.54} \mathrm{MnO}_{3}{ }^{[66,67]}$ 和 $\mathrm{La}_{0.81} \mathrm{Sr}_{0.19} \mathrm{MnO}_{3}{ }^{[68]}$ 中 的磁畴随温度发生的转变与交互作用, 以及磁畴在 外磁场作用下的转变. 指出 $\mathrm{La}_{0.81} \mathrm{Sr}_{0.19} \mathrm{MnO}_{3}$ 在室温 293 K下铁磁相和顺磁相共存, 且有闭合磁畴, 降低 温度完全转变成铁磁相, 升高温度变为顺磁相, 在 外磁场作用下, 分离的两个闭合磁畴会连通. 图 18 给出了 $\mathrm{La}_{0.46} \mathrm{Sr}_{0.54} \mathrm{MnO}_{3}$ 从反铁磁相到铁磁相, 铁磁 和反铁磁相磁畴共存结构以及穿过磁畴中心的相位 变化曲线. Yoo指出随着温度的升高, 闭合磁畴逐渐
缩小, 稍大点的磁畴逐渐分出两个小磁畴中心, 且两个磁畴的方向都是逆时针方向, 温度降低到室 温磁畴又恢复到原来的状态. Murakami给出了低温下 反铁磁相到铁磁相的转变, $202 \mathrm{~K} 反$ 铁磁和铁磁相共存, 随着温度的升高(从 202 到 $223 \mathrm{~K}$ ), 反铁磁逐渐向铁磁 相转变, 且在整个转变过程中磁畴都是闭合的. 继 $\mathrm{La}_{0.25} \mathrm{Pr}_{0.375} \mathrm{Ca}_{0.375} \mathrm{MnO}_{3}$ 中发现存在电荷有序和铁磁畴 共存之后, Loudon 等人 ${ }^{[69]}$ 也对 $\mathrm{La}_{0.5} \mathrm{Ca}_{0.5} \mathrm{MnO}_{3}$ 铁磁相 和电荷有序相进行了研究, 指出了铁磁相和电荷有 序化的共存现象.

Murakami 等人 ${ }^{[70,71]}$ 研究了 $\mathrm{Ni}_{51} \mathrm{Fe}_{22} \mathrm{Ga}_{27}$ 铁磁形 状记忆合金中的磁畴分布. 图 19 表明, 随着温度降 低内部磁畴有明显变化, 是由在冷却过程中磁的不 稳定性引起的, 而不是作为马氏体的前驱体或者中 间相形成的结构调制.

\section{3 其他材料}

电子全息的发展和应用正在不断地扩大, 除了 磁性材料、半导体材料和纳米材料之外, 还有一些电 子全息涉及相对较少, 但正在不断发展且具有深远 意义的材料, 如铁电材料的极化和调制结构等一系 列人们感兴趣的材料.

Zhang 等人 ${ }^{[74,75]}$ 首先采用电子全息研究了铁电 畴结构, 给出了铁电 $\mathrm{BaTiO}_{3}$ 中 $90^{\circ}$ 畴的电子全息图, 根据条纹间距和条纹的位移计算出相位的大小以及 畴的宽度, 尽管在理论上有些争论, 但是指出了电子 全息研究铁电畴的可行性. 随后Lichte ${ }^{[76]}, \mathrm{Cao}^{[77]}$ 和 Honda ${ }^{[78,79]}$ 等人分别对铁电畴和极化的问题进行了理 论和实验研究, 我们也对类似畴结构进行了研究, 却 没有得到类似的结果, 有待进一步的研究和探讨. 


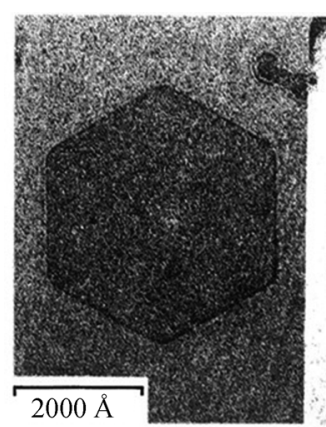

(a)

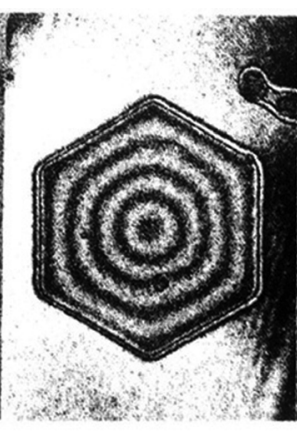

(b)

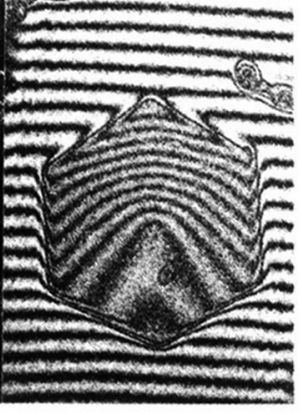

(c)

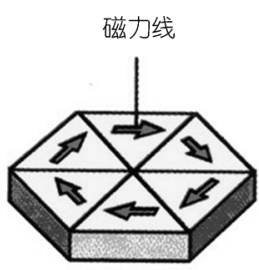

(d)

图 17 钴纳米颗粒全息图及内部磁力线分布

(a) 重构出的振幅图，颗粒内部没有衬度变化; (b) 同轴干涉形成的 2 倍放大的全息相位图;

(c) 2 倍放大的离轴全息相位图; (d) 磁力线分布示意图

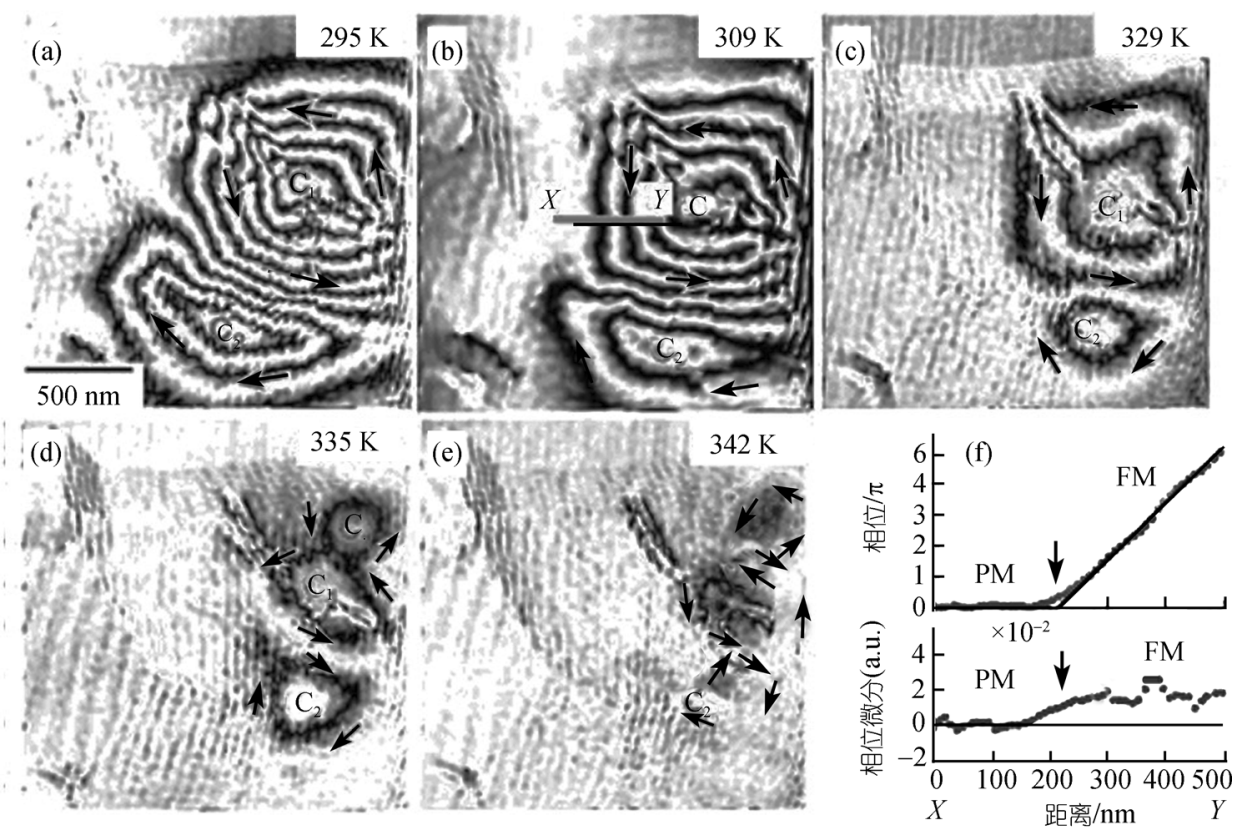

图 $18 \mathrm{La}_{0.46} \mathrm{Sr}_{0.54} \mathrm{MnO}_{3}$ 中混合相(铁磁到顺磁)的磁畴随温度变化

(a) (e) 重构出的相位图，箭头表示磁通量方向; (f) 上面的曲线是 $X-Y$ 方向的相位移, 下面的曲线是相位移的微分 

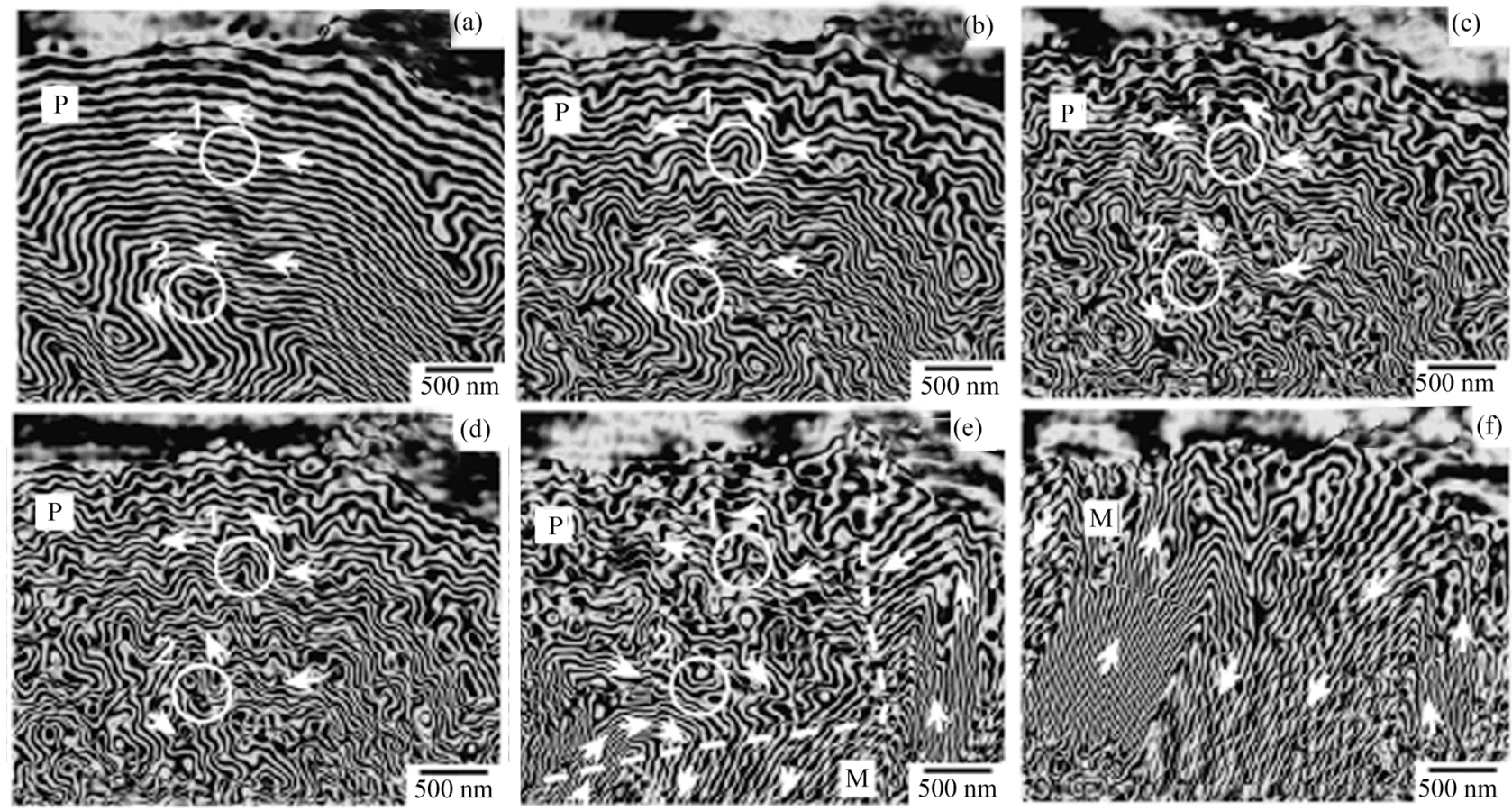

图 $19 \mathrm{Ni}_{51} \mathrm{Fe}_{22} \mathrm{Ga}_{27}$ 铁磁形状记忆合金中磁畴结构随温度母相到马氏体相的变化

(a) $296 \mathrm{~K}$; (b) $234 \mathrm{~K}$; (c) $193 \mathrm{~K}$; (d) $167 \mathrm{~K}$; (e) $162 \mathrm{~K}$; (f) $121 \mathrm{~K}$. P 和 M 分别代表母相和马氏体相

Schweda等人 ${ }^{[80]}$ 采用电子全息初步研究了 $\mathrm{Zr}_{4} \mathrm{ON}_{3} \mathrm{~F}_{5}$ 调制结构中由于层错导致的第二类调制结构, 指出电子 全息是能够研究金属中的层错和超结构中的位错, 电子束加热过程中的结构变化以及所谓无公度相的 形成等复杂结构, 还有待进一步的发展. Schofield等 人 ${ }^{[81]}$ 对 $\mathrm{Bi}_{2} \mathrm{Sr}_{2} \mathrm{CaCu}_{2} \mathrm{O}_{8+\delta}$ 中的BiO-BiO之间的电势和电 荷分布进行了实际测量和理论模拟, 讨论了物镜球 差和欠焦量对电子全息的影响.

\section{3 结论}

随着场发射电子显微镜及其相应部件的发展, 电子全息获得了广泛的发展. 与普通高分辨成像不 同的是，电子全息可以获得穿过物体的电子波的相 位变化，使电子全息在不同领域获得应用. 本文综述 了电子全息从 20 世纪 70 年代以来的发展、形成的基 本原理以及在材料研究中的一些应用, 系统论述了 一系列材料研究中的典型问题, 例如磁性材料中的 磁畴、磁分布和磁转变等, 非磁性材料中样品的平均 内电势分布、晶体管和异质结内电势、电荷密度分布、 缺陷结构、位错密度分布等, 铁电材料的极化以及有 关材料的三维形态信息. 近年来，电子全息的研究热 点集中在磁性材料中的磁畴研究，原子尺度的高分
辨电子全息研究，电子全息在材料内电势和样品厚 度方面的研究尽管很多, 但还没有形成一个简单实 用的方法，因此界面势垒研究还不能完全定量. 另外 如何用电子全息把材料内部电荷运动直观表达出来 也是下一步研究的重要方向.

致谢 感谢王岩国老师和段晓峰老师在实验上的帮助，感 谢吕惠宾和孙继荣老师提供样品. 本工作为国家自然科学 基金(批准号: 10225415)资助项目

\section{参考文献}

1 Cowley J M. Twenty forms of electron holography. Ultramicroscopy, 1992, 41: 335-348[DOI]

2 Cai J, Ponce F A. Study of the charge distribution across interface in $\mathrm{GaN} / \mathrm{InGaN} / \mathrm{GaN}$ single quantum wells using electron holography. J Appl Phys, 2002, 91(12): 9856-9862 [DOI]

3 Twitchett A C, Dunin-Borkowski R E, Broom R F, et al. Quantitative electron holography of biased semiconductor devices. J Phys: Condens Matter, 2004, 16: S181—S192[DOI]

4 Harada K, Akashi T, Togawa Y, et al. Optical system for double-biprism electron holography. J Electr Microsc, 2005, 54(1): 19-27[DOI]

5 Völkl E, Lehmann M. Introduction to Electron Holography. New York: Kluwer/Plenum, 1999. 125

6 Lehmann $\mathrm{M}$, Lichte $H$. Electron holographic material analysis at atomic dimensions. Crys Res Technol, 2005, 40(1/2): 149—160[DOI] 
7 McCartney M R, Smith D J, Hull R, et al. Direct observation of potential distribution across $\mathrm{Si} / \mathrm{Si}$ p-n junctions using off-axis electron holography. Appl Phys Lett, 1994, 65(20): 2603-2605[DOI]

8 Smith D J, McCartney M R. Introduction to Electron Holography. New York: Kluwer/Plenum, 1999. 87

9 Völkl E, Allard L F, Datye A, et al. Advanced electron holography: A new algorithm for image processing and a standardized quality test for the FEG electron microscope. Ultramicroscopy, 1995, 58: $97-103$ [DOI]

10 de Ruijter W J, Weiss J K. Methods to measure properties of slow-scan CCD cameras for electron detection. Rev Sci Instrum, 1992, 63: 4314-4321[DOI]

11 Fu Q, Lichte H, Völkl E. Correction of aberration of an electron microscopy by mean of electron holography. Phys Rev Lett, 1991, 67(17): 2319-2322[DOI]

12 Lehmann M. Determination and correction of the coherent wave aberration from a single off-axis electron hologram by means of a genetic algorithm. Ultramicroscopy, 2000, 85: 165-182[DOI]

13 Gajdardziska-Josifovska M, McCartney M R, de Ruijter W J, et al. Accurate measurement of mean inner potential of crystal wedges using digital electron holograms. Ultramicroscopy, 1993, 50: 285-289[DOI]

14 Li J, McCartney M R, Dunin-Borkowski R E, et al. Determination of mean inner potential of germanium using off-axis electron holography. Acta Cryst, 1999, A55: 652-658

15 Kruse P, Rosenauer A, Gerthsen D. Determination of the mean inner potential in III-V semiconductors by electron holography. U1tramicroscopy, 2003, 96: 11-16[DOI]

16 McCartney M R, Gajdardziska-Josifovska M. Absolute measurement of normalized thickness, $t / \lambda_{i}$, from off-axis electron holography. Ultramicroscopy, 1994, 53: 283-289[DOI]

17 Allard L F, Völkl E, Carim A, et al. Morphology and crystallography of nano-particulates revealed by electron holography. Nano Mater, 1996, 7: 137-146[DOI]

18 Tonomura A, Matsuda $\mathrm{T}$, Tanabe H, et al. Electron holography technique for investigating thin ferromagnetic films. Phys Rev B, 1982, 25: 6799-6804[DOI]

19 Matsuda T, Tonomura A, Suzuki R, et al. Observation of microscopic distribution of magnetic fields by electron holography. J Appl Phys, 1982, 53(8): 5444-5446[DOI]

20 Osakabe N, Yoshida K, Horiuchi Y, et al. Observation of recorded magnetization pattern by electron holography. Appl Phys Lett, 1983, 42: 746-748[DOI]

21 Tonomura A, Matsuda T, Endo J. Holographic interference electron microscopy for determining specimen magnetic structure and thickness distribution. Phys Rev B, 1986, 34: 3397-3402[DOI]

22 Frabboni S, Matteucci G, Pozzi G. Electron holographic observations of the electrostatic field associated with thin reverse-biased p-n junctions. Phys Rev Lett, 1985, 55(11): 2196-2199[DOI]

23 Ravikumar V, Rodrigues R P, Dravid V P. Direct imaging of spatially varying potential and charge across internal interfaces in solids. Phys Rev Lett, 1995, 75(22): 4063-4066[DOI]

24 Johnson K D, Dravid V P. Grain boundary barrier breakdown in niobium donor doped strontium titanate using in situ electron holography. Appl Phys Lett, 1999, 74(4): 621-623[DOI]

25 Weiss J K, de Ruijter W J, Gajdardziska-Josifovska M, et al. Application of electron holography to study of interfaces. Ultramicroscopy, 1993, 50: 301-311[DOI]

26 Gajdardziska-Josifovska M. Off-axis electron holography of hetero-interfaces. Interface Sci, 1995, 2: 425-440

27 Formanek P, Kittler M. Direct evidence of internal Schottky barriers at $\mathrm{NiSi}_{2}$ precipitates in silicon by electron holography. $\mathrm{J}$ Appl Phys, 2005, 97: 063707 [DOI]

28 Elfwing M, Olsson E. Electron holography study of active interfaces in zinc oxide varistor materials. J Appl Phys, 2002, 92: $5272-5280[\mathrm{DOI}]$

29 Rau W D, Schwander P, Baumann F H, et al. Two-dimensional mapping of the electrostatic potential in transistors by electron holography. Phys Rev Lett, 1999, 82: 2614-2617[DOI]

30 Wang Y C, Chou T M, Libera M, et al. Transmission electron holography silicon nanospheres with surface oxide layers. Appl Phys Lett, 1997, 70(10): 1296-1298[DOI]

31 Wang Z G, Sasaki K, Naoko K, et al. Examination of electrostatic potential distribution across an implanted p-n junction by electron holography. J Electron Microsc, 2001, 50(6): 479-484[DOI]

$32 \mathrm{Wu} \mathrm{L}$, Schofield M A, Zhu Y, et al. A unique determination of boundary condition in quantitative electron diffraction: Application to accurate measurements of mean inner potentials. Ultramicroscopy, 2004, 98: 135-143[DOI]

33 Elfwing M, Olsson E. Electron holography study of active interfaces in zinc oxide varistor materials. J Appl Phys, 2002, 92(9): $5272-5280[\mathrm{DOI}]$

34 Müller E, Kruse P, Gerthsen D, et al. Measurement of mean inner potential of $\mathrm{ZnO}$ nanorods by transmission electron holography. Appl Phys Lett, 2005, 86(15): 154108[DOI]

35 Yada K, Shibata K, Hibi T. A high resolution electron interference microscope and its application to the measurement of mean inner potential. J Electron Microscopy, 1973, 22: 223-230

36 Cherns D, Barnard J, Ponce F A. Measurement of the piezoelectric field across strained InGaN/GaN layers by electron holography. Solid State Commun, 1999, 111: 281-285[DOI]

37 McCartney M R, Ponce F A, Cai J. Mapping electrostatic across an AlGaN/InGaN/AlGaN diode electron holography. Appl Phys Lett, 2000, 76(21): 3055-3057[DOI]

38 Ichikawa S, Akita T, Okazaki K, et al. Mean inner potential of nanostructure noble metal catalysts $-\mathrm{Pt} / \mathrm{TiO}_{2}$ catalyst. Contin Nanoph Nanostruct Mater, 2004, 788: 443-448

39 Wang Y G, Liu H R, Yang Q B, et al. Determination of mean inner potential by electron holography along with electron dynamic simulation. Chin Phys Lett, 2003, 20(12): 2214-2217[DOI]

40 Lin X W, Dravid V P. Mapping the potential of graphite nanotubes with electron holography. Appl Phys Lett, 1996, 69(7): 1014- 


\section{6[DOI]}

41 Houben L, Luysberg M, Brammer T. Illumination effects in holographic imaging of the electrostatic potential of defects and pn junctions in transmission electron microscopy. Phys Rev B, 2004, 70(16): 165313[DOI]

42 Beleggia M, Fazzini P F, Merli P G, et al. Influence of charged oxide layers on TEM imaging of reverse-biased $p-n$ junctions. Phys Rev B, 2003, 67: 045328[DOI]

43 Twitchett A C, Dunin-Borkowski R E, Hallifax R J, et al. Off-axis electron holography of unbiased and reverse-biased focused ion beam milled Si p-n junctions. Microsc Microanal, 2005, 11: $1-13$ [DOI]

44 McCartney M R, Gribelyuk M A, Li J, et al. Quantitative analysis of one-dimensional dopant profile by electron holography. Appl Phys Lett, 2002, 80(17): 3213-3215[DOI]

45 Wang Z G, Hirayama T, Sasaki K, et al. Electron holographic characterization of electrostatic potential distributions in a transistor sample fabricated by focused ion beam. Appl Phys Lett, 2002, 80(2): 246-248[DOI]

46 Gribelyuk M A, McCartney M R, Li J, et al. Mapping of electrostatic potential in deep submicron CMOS devices by electron holography. Phys Rev Lett, 2002, 89(2): 025502[DOI]

47 Twitchett A C, Dunin-Borkowski R E, Midgley P A. Quantitative electron holography of biased semiconductor devices. Phys Rev Lett, 2002, 88(10): 238302[DOI]

48 Twitchett A C, Dunin-Borkowski R E, Hallifax R J. Off-axis electron holography of electrostatic potentials in unbiased and reverse biased focused ion beam milled semiconductor devices. J Microsc, 2004, 214: 287-296[DOI]

49 Cherns D, Jiao C G. Electron holography studies of the charge on dislocations in GaN. Phys Rev Lett, 2001, 87(20): 205504[DOI]

50 Jiao C G, Cherns D. Investigation of the charge on threading edge dislocations in GaN by electron holography. J Electron Microsc, 2002, 51(2): 105-112[DOI]

51 Cai, J, Ponce F A, Tanaka S, et al. Mapping the internal potential across $\mathrm{GaN} / \mathrm{AlGaN}$ heterostructures by electron holography. Phys Stat Solid A, 2001, 188: 833-837[DOI]

52 Cai J, Ponce F A. Study of charge distribution across interface in $\mathrm{GaN} / \mathrm{InGaN} / \mathrm{GaN}$ single quantum wells using electron holography. J Appl Phys, 2002, 91(12): 9856-9862[DOI]

53 Stevens M, Bell A, McCartney M R, et al. Effect of layer thickness on the electrostatic potential in InGaN quantum well. Appl Phys Lett, 2004, 85(20): 4651—4653[DOI]

54 Tian H F, Yu H C, Zhu X H, et al. Off-axis electron holography and microstructure of $\mathrm{Ba}_{0.5} \mathrm{Sr}_{0.5} \mathrm{TiO}_{3}$ thin films on $\mathrm{LaAlO}_{3}$. Phys Rev B, 2005, 71: 115419[DOI]

55 Tian H F, Sun J R, Lü H B, et al. Electrostatic potential in manganite-based heterojunctions by electron holography. Appl Phys Lett, 2005, 87(16): 164102[DOI]

56 Xu Q Y, Wang Y G, Zhang Z, et al. Characterization of the asymmetrical barrier potentials in $\mathrm{CoFe} / \mathrm{AlO}_{x} / \mathrm{Co}$ magnetic tunneling junction by electron holography. J Magn Magn Mater, 2003, 263: $83-87$ [DOI]

57 Shen F, Zhu T, Xiang X H, et al. Observation of the barrier structure in magnetic tunnel junctions using high-resolution electron microscopy and electron holography. Appl Phys Lett, 2003, 83: $5482-5484[\mathrm{DOI}]$

58 Dunin-Borkowski R E, McCartney M R, Kardynal B, et al. Off-axis electron holography of exchange-biased $\mathrm{CoFe} / \mathrm{FeMn}$ patterned nanostructures. J Appl Phys, 2001, 90: 2899-2902[DOI]

59 Dunin-Borkowski R E, McCartney M R, Kardynal B, et al. Off-axis electron holography of patterned magnetic nanostructures. J Microsc, 2000, 200: 187-205[DOI]

60 Dunin-Borkowski R E, McCartney M R, Kardynal B, et al. Magnetic interaction within patterned cobalt nanostructures using off-axis electron holography. J Appl Phys, 1998, 84(1): $374-378[\mathrm{DOI}]$

61 Shindo D, Park Y G, Gao Y H, et al. Electron holography of Fe-based nanocrystalline magnetic materials (invited). J Appl Phys, 2004, 95: 6521-6526[DOI]

62 Hirayama T, Ru Q, Tanji T, et al. Observation of magnetic-domain states of barium ferrite particles by electron holography. Appl Phys Lett, 1993, 63: 418-420[DOI]

63 Harada K, Beleggia M, Endo J, et al. Profile structure of magnetic flux lines in type-II superconductor from a rectangular electron hologram. J Electron Microsc, 2003, 52(4): 369-373[DOI]

64 Hasegawa S, Matsuda T, Endo J, et al. Magnetic flux quanta in superconducting films observed by electron holography and digital phase analysis. Phys Rev B, 1991, 43(10): 7631-7650[DOI]

65 Bonevich J E, Harada K, Matsuda T, et al. Electron holography observation of vortex lattices in a superconductor. Phys Rev Lett, 1993, 70(19): 2952—2955[DOI]

66 Yoo J H, Murakami Y, Shindo D, et al. Behavior of magnetic domains in $\mathrm{La}_{0.46} \mathrm{Sr}_{0.54} \mathrm{MnO}_{3}$ during the ferromagnetic phase transformation studied by electron holography. Phys Rev B, 2002, 66: $212406[\mathrm{DOI}]$

67 Murakami Y, Yoo J H, Shindo D, et al. Magnetization distribution in the mixed-phase state of hole-doped manganites. Nature, 2003, 423: 965 [DOI]

68 Yoo J H, Murakami Y, Shindo D, et al. Interaction of separated ferromagnetic domains in a hole-doped manganite achieved by a magnetic field. Phys Rev Lett, 2004, 93: 047204[DOI]

69 Loudon J C, Mathur N D, Midgley P A, et al. Charge-ordered ferromagnetic phase in $\mathrm{La}_{0.5} \mathrm{Ca}_{0.5} \mathrm{MnO}_{3}$. Nature, 2003, 420: 797[DOI]

70 Murakami Y, Shindo D, Oikawa K, et al. Magnetic domain structure in a ferromagnetic shape memory alloy $\mathrm{Ni}_{51} \mathrm{Fe}_{22} \mathrm{Ga}_{27}$ studied by electron holography and Lorentz microscopy. Appl Phys Lett, 2003, 82: 3695-3697[DOI]

71 Murakami Y, Shindo D, Oikawa K, et al. Microstructural change near the martensitic transformation in a ferromagnetic shape memory alloy $\mathrm{Ni}_{51} \mathrm{Fe}_{22} \mathrm{Ga}_{27}$ studied by electron holography. Appl Phys Lett, 2004, 85: 6170-6172[DOI] 
72 Bonevich J E, Rau W D, Lichte H, et al. Introduction to Electron Holography. New York: Kluwer/Plenum, 1999. 153-200

73 Tonomura A, Matsuda T, Endo J, et al. Holographic interference electron microscopy for determining specimen magnetic structure and thickness distribution. Phys Rev B, 1986, 34: 3397-3402[DOI]

74 Zhang X, Hashimoto T, Joy D C. Electron holography study of ferroelectric domain walls. Appl Phys Lett, 1992, 60(6): $784-786[\mathrm{DOI}$

75 Zhang X, Joy D C, Zhang Y, et al. Electron holography techniques for study of ferroelectric domain walls. Ultramicroscopy, 1993, 51: $21-30[\mathrm{DOI}]$

76 Lichte H, Reibold M, Brand K, et al. Ferroelectric electron holography. Ultramicroscopy, 2002, 93: 199-212[DOI]

77 Cao W W, Randall C. Theory on the fringe patterns in the study of ferroelectric domain walls using electron holography. Solid State Commun, 1993, 86(7): 435-439[DOI]

78 Honda K. Direct observation of potential distribution across ferro- electric capacitor using off-axis electron holography. In: Proceedings of the Eleventh IEEE International Symposium on Applications of Ferroelectrics. Montreux Switzerland: The Institute of Electrical and Electronic Engineers Ultrasonic, Ferroelectrics and Frequency Control Society, 1998. 463-466

79 Honda K. Direct observation of potential distribution across $\mathrm{PbZr}_{x} \mathrm{Ti}_{1-x} \mathrm{O}_{3}$ thin films using off-axis electron holography. FUJITSU Sci Tech J, 2002, 38(1): 64-68

80 Schweda E, Strähle J, Lichte H, et al. Combination of high-resolution electron imaging and holography in the investigation of the modulated structure of $\mathrm{Zr}_{4} \mathrm{ON}_{3} \mathrm{~F}_{5}$. Ultramicroscopy, 1994, 53: $53-62$ [DOI]

81 Schofield M A, Wu L, Zhu Y. Quantitative measurement of grain boundary potentials on the nanoscale by off-axis electron holography. Phys Rev B, 2003, 67: 224512[DOI]

(2005-06-18 收稿, 2006-02-16 接受) 